\title{
Heat and Mass Transfer from an Inverted Cone in a Porous Medium with Cross-Diffusion Effects
}

\author{
Faiz GA Awad, Precious Sibanda and Mahesha Narayana \\ School of Mathematical Sciences, University of KwaZulu-Natal \\ South Africa
}

\section{Introduction}

The study of double-diffusive convection has received considerable attention during the last several decades since this occurs in a wide range of natural settings. The origins of these studies can be traced to oceanography when hot salty water lies over cold fresh water of a higher density resulting in double-diffusive instabilities known as "salt-fingers," Stern (35; 36). Typical technological motivations for the study of double-diffusive convection range from such diverse fields as the migration of moisture through air contained in fibrous insulations, grain storage systems, the dispersion of contaminants through water-saturated soil, crystal growth and the underground disposal of nuclear wastes. Double-diffusive convection has also been cited as being of particular relevance in the modeling of solar ponds (Akbarzadeh and Manins (1)) and magma chambers (Fernando and Brandt (12)).

Double-diffusive convection problems have been investigated by, among others, Nield (28) Baines and Gill (3), Guo et al. (14), Khanafer and Vafai (17), Sunil et al. (37) and Gaikwad et al. (13). Studies have been carried out on horizontal, inclined and vertical surfaces in a porous medium by, among others, Cheng (9; 10), Nield and Bejan (29) and Ingham and Pop (32). $\mathrm{Na}$ and Chiou (24) presented the problem of laminar natural convection in Newtonian fluids over the frustum of a cone while Lai (18) investigated the heat and mass transfer by natural convection from a horizontal line source in saturated porous medium. Natural convection over a vertical wavy cone has been investigated by Pop and $\mathrm{Na}$ (33). Nakyam and Hussain (25) studied the combined heat and mass transfer by natural convection in a porous medium by integral methods.

Chamkha and Khaled (4) studied the hydromagnetic heat and mass transfer by mixed convection from a vertical plate embedded in a uniform porous medium. Chamkha (5) investigated the coupled heat and mass transfer by natural convection of Newtonian fluids about a truncated cone in the presence of magnetic field and radiation effects and Yih (38) examined the effect of radiation in convective flow over a cone. Cheng (6) used an integral approach to study the heat and mass transfer by natural convection from truncated cones in porous media with variable wall temperature and concentration. Khanafer and Vafai (17) studied the double-diffusive convection in a lid-driven enclosure filled with a fluid-saturated porous medium. Mortimer and Eyring (22) used an elementary transition state approach to obtain a simple model for Soret and Dufour effects in thermodynamically ideal mixtures of substances with molecules of nearly equal size. In their model the flow of heat in the Dufour effect was identified as the transport of the enthalpy change of activation as molecules diffuse. 
The results were found to fit the Onsager reciprocal relationship (Onsager, (30)). Alam et al. (2) investigated the Dufour and Soret effects on steady combined free-forced convective and mass transfer flow past a semi-infinite vertical flat plate of hydrogen-air mixtures. They used the fourth order Runge-Kutta method to solve the governing equations of motion. Their study showed that the Dufour and Soret effects should not be neglected. Mansour et al. (21) studied the effects of a chemical reaction and thermal stratification on MHD free convective heat and mass transfer over a vertical stretching surface embedded in a porous media with Soret and Dufour effects. Narayana and Murthy (26) examined the Soret and Dufour effects on free convection heat and mass transfer from a horizontal flat plate in a Darcy porous medium.

The effects of the Soret and Dufour parameters on free convection along a vertical wavy surface in a Newtonian fluid saturated Darcy porous medium has been investigated by Narayana and Sibanda (27). Their study showed that in both the aiding and opposing buoyancy cases increasing the Soret parameter leads to a reduction in the axial mass transfer coefficient. They further showed that the effect of the Dufour parameter is to increase the heat transfer coefficient at the surface. On the other hand, the mass transfer coefficient increased with the Dufour parameter only up to a certain critical value of the Soret parameter. Beyond this critical value, the mass transfer coefficient decreased with increasing Dufour parameter values.

The thermophoresis effect on a vertical plate embedded in a non-Darcy porous medium with suction and injection and subject to Dufour and Soret effects was investigated by Partha (31). The findings in this study underlined the importance of the Dufour, Soret and dispersion parameters on heat and mass transfer. The results showed that the Soret effect is influential in increasing the concentration distribution in both aiding as well as opposing buoyancy cases. Cheng (8) studied the Dufour and Soret effects on heat and mass transfer over a downward-pointing vertical cone embedded in a porous medium saturated with a Newtonian fluid and constant wall temperature and concentration.

In this work we investigate heat and mass transfer from an inverted smooth and a wavy cone in porous media. In the case of the smooth cone we extend the work of Murthy and Singh (23) and El-Amin (11) to include cross-diffusion effects.

As with most problems in science and engineering, the equations that describe double-diffusive convection from an inverted cone in a porous medium are highly nonlinear and do not have closed form solutions. For the smooth cone, the equations are solved used the successive linearisation method (see Makukula et al. (19; 20)) which combines a non-perturbation technique with the Chebyshev spectral collocation method to produce an algorithm that is numerically accurate. The accuracy and robustness of the linearisation method is proved by using the Matlab bvp4c numerical routine and a shooting method to solve the equations. For the wavy cone, the governing nonlinear partial differential equations are solved using the well known Keller-box method.

\section{Flow over a smooth cone in porous medium}

Consider the problem of double-diffusive convection flow over inverted cone with half-angle $\Omega$, embedded in a saturated non-Darcy porous medium as shown in Figure 1. The origin of the coordinate system is at the vertex of the cone. The $x$-axis measures the distance along the surface of the cone and the $y$-axis measures the distance outward and normal to the surface of the cone. The surface of the cone is subject to a non-uniform temperature $T_{w}>T_{\infty}$ where $T_{\infty}$ is the temperature far from the cone surface. The solute concentration varies from $C_{w}$ on the surface of the inverted cone to a lower concentration $C_{\infty}$ in the ambient fluid. The solid 
and fluid phases are assumed to be in local thermal equilibrium. The governing equations for such a flow are (see Yih (38), Cheng (8), Murthy (23), El-Amin (11));

$$
\begin{aligned}
& \frac{\partial}{\partial x}(r u)+\frac{\partial}{\partial y}(r v)=0, \\
& \frac{\partial u}{\partial y}+\frac{c \sqrt{K}}{v} \frac{\partial u^{2}}{\partial y}=\frac{K g \beta \cos \Omega}{v}\left(\frac{\partial T}{\partial y}+\frac{\beta^{*}}{\beta} \frac{\partial C}{\partial y}\right), \\
& u \frac{\partial T}{\partial x}+v \frac{\partial T}{\partial y}=\frac{\partial}{\partial y}\left(\alpha_{y} \frac{\partial T}{\partial y}\right)+\frac{D k_{T}}{c_{s} c_{p}} \frac{\partial^{2} C}{\partial y^{2}}, \\
& u \frac{\partial C}{\partial x}+v \frac{\partial C}{\partial y}=\frac{\partial}{\partial y}\left(D_{y} \frac{\partial C}{\partial y}\right)+\frac{D k_{T}}{c_{s} c_{p}} \frac{\partial^{2} T}{\partial y^{2}},
\end{aligned}
$$

where for a thin boundary layer, $r=x \sin \Omega$, g is the acceleration due to gravity, $c$ is an empirical constant, $K$ is the permeability, $v$ is kinematic viscosity of the fluid, respectively, $\beta$ and $\beta^{*}$ are the thermal expansion and the concentration expansion coefficients, $\alpha_{y}$ and $D_{y}$ are the effective thermal and mass diffusivities of the saturated porous medium defined by $\alpha_{y}=\alpha+\gamma d u$ and $D_{y}=D+\xi d u$, respectively, $\gamma$ and $\xi$ are coefficients of thermal and solutal dispersions, respectively, $\alpha$ and $D$ are constant thermal and molecular diffusivities, $k_{T}$ is the thermal diffusion ratio, $c_{S}$ is concentration susceptibility and $c_{p}$ is the specific heat at constant pressure. We assume a nonlinear power-law for temperature and concentration

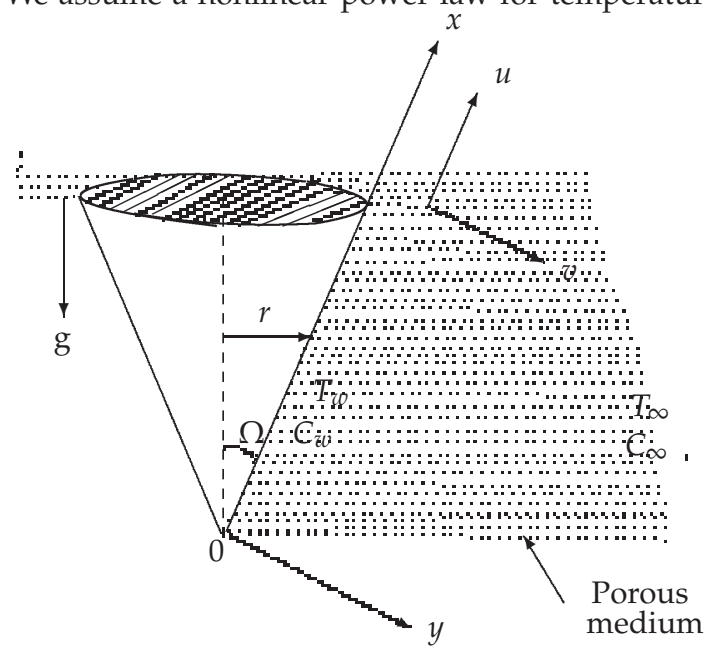

Fig. 1. Inverted smooth cone in a porous medium

variations within the fluid so that the boundary conditions are

$$
\begin{aligned}
& v=0, u=0, T=T_{w}=T_{\infty}+A x^{n}, C=C_{w}=C_{\infty}+B x^{n} \quad \text { on } \quad y=0, x \geq 0 \\
& u=0, \quad T=T_{\infty}, \quad C=C_{\infty} \quad \text { as } y \rightarrow \infty,
\end{aligned}
$$

where $A, B>0$ are constants and $n$ is the power-law index. The subscripts $w, \infty$ refer to the cone surface and ambient conditions respectively. We introduce the similarity variables

$$
\eta=\frac{y}{x} R a_{x}^{\frac{1}{2}}, \quad \psi=\alpha r R a_{x} \frac{1}{2} f(\eta), \quad \theta(\eta)=\frac{T-T_{\infty}}{T_{w}-T_{\infty}}, \quad \phi(\eta)=\frac{C-C_{\infty}}{C_{w}-C_{\infty}},
$$


where $\psi$ is the stream function and $R a_{x}$ is the Rayleigh number defined by:

$$
u=\frac{1}{r} \frac{\partial \psi}{\partial y}, \quad v=-\frac{1}{r} \frac{\partial \psi}{\partial x} \quad \text { and } \quad R a_{x}=\frac{\mathrm{g} \beta K \cos \Omega\left(T_{w}-T_{\infty}\right) x}{\alpha v} .
$$

The dimensionless momentum, energy and concentration equations become

$$
\begin{aligned}
& f^{\prime \prime}+2 \lambda f^{\prime} f^{\prime \prime}-\theta^{\prime}-N \phi^{\prime}=0, \\
& \theta^{\prime \prime}+\frac{n+3}{2} f \theta^{\prime}-n f^{\prime} \theta+R a_{\gamma}\left(f^{\prime \prime} \theta^{\prime}+f^{\prime} \theta^{\prime \prime}\right)+D_{f} \phi^{\prime \prime}=0, \\
& \frac{1}{L e} \phi^{\prime \prime}+\frac{n+3}{2} f \phi^{\prime}-n f^{\prime} \phi+R a_{\xi}\left(f^{\prime \prime} \phi^{\prime}+f^{\prime} \phi^{\prime \prime}\right)+S_{r} \theta^{\prime \prime}=0,
\end{aligned}
$$

subject to the boundary conditions

$$
\begin{aligned}
& f=0, \quad \theta=1, \quad \phi=1 \quad \text { on } \quad \eta=0, \\
& f^{\prime}=0, \quad \theta=0, \quad \phi=0 \quad \text { on } \quad \eta \rightarrow \infty .
\end{aligned}
$$

where primes denote differentiation with respect to $\eta$. The important thermo-physical parameters are the buoyancy ratio $N$ (where $N>0$ represents aiding buoyancy and $N<0$ represents the opposing buoyancy), the Dufour parameter $D_{f}$, the Soret parameter $S_{r}$, the pore depended Rayleigh number $R a_{d}$ and the Lewis number Le. These are defined as

$$
\begin{aligned}
& N=\frac{\beta^{*}}{\beta} \frac{C_{w}-C_{\infty}}{T_{w}-T_{\infty}}, D_{f}=\frac{D k_{T}}{C_{s} c_{p}} \frac{C_{w}-C_{\infty}}{\alpha\left(T_{w}-T_{\infty}\right)}, S_{r}=\frac{D k_{T}}{c_{s} c_{p}} \frac{\alpha\left(T_{w}-T_{\infty}\right)}{C_{w}-C_{\infty}}, \\
& R a_{d}=\frac{g \beta K \cos (\Omega)\left(T_{w}-T_{\infty}\right) d}{\alpha v}, L e=\frac{\alpha}{D}, \hat{\sigma}=\frac{C \sqrt{K} \alpha}{v d},
\end{aligned}
$$

where $R a_{\gamma}=\gamma R a_{d}, R a_{\xi}=\xi R a_{d}$ represent the thermal and solutal dispersions respectively, $\lambda=\hat{\sigma} R a_{d}$ and $\hat{\sigma}$ is an inertial parameter. The parameters of engineering interest in heat and mass problems are the local Nusselt number $N u_{x}$ and the local Sherwood number $S h_{x}$. These parameters characterize the surface heat and mass transfer rates respectively. The local heat and mass transfer rates from the surface of the cone are characterized by the Nusselt and Sherwood numbers respectively where

$$
N u_{x}=-R a_{x}^{\frac{1}{2}}\left[1+R a_{\gamma} f^{\prime}(0)\right] \theta^{\prime}(0) \quad \text { and } \quad S h_{x}=-R a_{x}^{\frac{1}{2}}\left[1+R a_{\xi} f^{\prime}(0)\right] \phi^{\prime}(0) .
$$

\subsection{Method of solution}

To solve equations (9) - (12), the successive linearisation method (see Makukula et al. $(19 ; 20)$ ) was used. This assumes that the functions $f(\eta), \theta(\eta)$ and $\phi(\eta))$ may be expressed as

$$
\begin{aligned}
f(\eta) & =f_{i}(\eta)+\sum_{m=0}^{i-1} f_{m}(\eta), \\
\theta(\eta) & =\theta_{i}(\eta)+\sum_{m=0}^{i-1} \theta_{m}(\eta), \\
p h i(\eta) & =\phi_{i}(\eta)+\sum_{m=0}^{i-1} \phi_{m}(\eta),
\end{aligned}
$$


where $f_{i}, \theta_{i}, \phi_{i}(i=1,2,3, \ldots)$ are such that

$$
\lim _{i \rightarrow \infty} f_{i}=\lim _{i \rightarrow \infty} \theta_{i}=\lim _{i \rightarrow \infty} \phi_{i}=0 .
$$

The functions $f_{m}, \theta_{m}$ and $\phi_{m}(m \geq 1)$ are approximations that are obtained by recursively solving the linear parts of the equations that result from substituting (16) in equations (9) - (11). Using the above assumptions, nonlinear terms in $f_{i}, \theta_{i}, \phi_{i}$ and their corresponding derivatives are considered to be very small and therefore neglected.

Starting from the initial guesses

$$
f_{0}(\eta)=1-e^{-\eta}, \quad \theta_{0}(\eta)=e^{-\eta} \quad \text { and } \quad \phi_{0}(\eta)=e^{-\eta},
$$

which are chosen to satisfy boundary conditions (12), the subsequent solutions for $f_{i}, h_{i}, \theta_{i}$ $i \geq 1$ are obtained by successively solving the linearized form of the governing equations.

The linearized equations to be solved are

$$
\begin{aligned}
& a_{1, i-1} f_{i}^{\prime \prime}+a_{2, i-1} f_{i}^{\prime}-\theta_{i}^{\prime}-N \phi_{i}^{\prime}=r_{1, i-1}, \\
& b_{1, i-1} \theta_{i}^{\prime \prime}+b_{2, i-1} \theta_{i}^{\prime}+b_{3, i-1} \theta_{i}+b_{4, i-1} f_{i}^{\prime \prime}+b_{5, i-1} f_{i}^{\prime}+b_{6, i-1} f_{i}+D_{f} \phi_{i}^{\prime \prime}=r_{2, i-1}, \\
& c_{1, i-1} \phi_{i}^{\prime \prime}+c_{2, i-1} \phi_{i}^{\prime}+c_{3, i-1} \phi_{i}+c_{4, i-1} f_{i}^{\prime \prime}+c_{5, i-1} f_{i}^{\prime}+c_{6, i-1} f_{i}+\operatorname{Sr} \theta_{i}^{\prime \prime}=r_{3, i-1},
\end{aligned}
$$

subject to the boundary conditions

$$
f_{i}(0)=f_{i}^{\prime}(\infty)=0, \quad \theta_{i}(0)=\theta_{i}(\infty)=\phi_{i}(0)=\phi_{i}(\infty)=0 .
$$

The coefficient parameters $a_{k, i-1}, b_{k, i-1}, c_{k, i-1}(k=1,2, \ldots, 6), r_{j, i-1}(j=1,2,3)$ are given by

$$
\begin{aligned}
& a_{1, i-1}=1+2 \lambda \sum_{m=0}^{i-1} f_{m}^{\prime}, \quad a_{2, i-1}=2 \lambda \sum_{m=0}^{i-1} f_{m}^{\prime \prime}, \\
& b_{1, i-1}=1+R a_{\gamma} \sum_{m=0}^{i-1} f_{m}^{\prime}, \quad b_{2, i-1}=\frac{n+3}{2} \sum_{m=0}^{i-1} f_{m}+R a_{\gamma} \sum_{m=0}^{i-1} f_{m}^{\prime \prime}, \\
& b_{3, i-1}=-n \sum_{m=0}^{i-1} f_{m}^{\prime}, \quad b_{4, i-1}=R a_{\gamma} \sum_{m=0}^{i-1} \theta_{m}^{\prime}, \quad b_{5, i-1}=R a_{\gamma} \sum_{m=0}^{i-1} \theta_{m}^{\prime \prime}-n \sum_{m=0}^{i-1} \theta_{m}^{\prime}, \\
& b_{6, i-1}=\frac{n+3}{2} \sum_{m=0}^{i-1} \theta_{m}^{\prime}, \\
& c_{1, i-1}=\frac{1}{L e}+R a_{\xi} \sum_{m=0}^{i-1} f_{m}^{\prime}, \quad c_{2, i-1}=\frac{n+3}{2} \sum_{m=0}^{i-1} f_{m}+R a_{\xi} \sum_{m=0}^{i-1} f_{m}^{\prime \prime}, \\
& c_{3, i-1}=-n \sum_{m=0}^{i-1} f_{m}^{\prime}, \quad c_{4, i-1}=R a_{\xi} \sum_{m=0}^{i-1} \phi_{m}^{\prime}, \quad c_{5, i-1}=R a_{\xi} \sum_{m=0}^{i-1} \phi_{m}^{\prime \prime}-n \sum_{m=0}^{i-1} \phi_{m}^{\prime}, \\
& c_{6, i-1}=\frac{n+3}{2} \sum_{m=0}^{i-1} \phi_{m}^{\prime},
\end{aligned}
$$




$$
\begin{aligned}
r_{1, i-1}= & -\left[\sum_{m=0}^{i-1} f_{m}^{\prime \prime}+2 \lambda \sum_{m=0}^{i-1} f_{m}^{\prime} \sum_{m=0}^{i-1} f_{m}^{\prime \prime}-\sum_{m=0}^{i-1} h_{m}^{\prime}-N \sum_{m=0}^{i-1} g_{m}^{\prime}\right], \\
r_{2, i-1}= & -\left[\sum_{m=0}^{i-1} \theta_{m}^{\prime \prime}+D_{f} \sum_{m=0}^{i-1} \phi_{m}^{\prime \prime}+\frac{n+3}{2} \sum_{n=0}^{i-1} f_{m} \sum_{n=0}^{i-1} \theta_{n}^{\prime}-n \sum_{m=0}^{i-1} f_{m}^{\prime} \sum_{m=0}^{i-1} \theta_{m}\right. \\
& \left.+R a_{\gamma}\left(f_{m}^{\prime \prime} \sum_{m=0}^{i-1} \theta_{m}^{\prime}+f_{m}^{\prime} \sum_{m=0}^{i-1} \theta_{m}^{\prime \prime}\right)\right], \\
r_{3, i-1}=- & {\left[\frac{1}{L e} \sum_{m=0}^{i-1} \phi_{m}^{\prime \prime}+S_{r} \sum_{m=0}^{i-1} \phi_{m}^{\prime \prime}+\frac{n+3}{2} \sum_{m=0}^{i-1} f_{m} \sum_{m=0}^{i-1} \phi_{m}^{\prime}-n \sum_{m=0}^{i-1} f_{m}^{\prime} \sum_{m=0}^{i-1} \phi_{m}\right.} \\
+ & \left.R a_{\gamma}\left(f_{m}^{\prime \prime} \sum_{m=0}^{i-1} g_{m}^{\prime}+f_{m}^{\prime} \sum_{m=0}^{i-1} \phi_{m}^{\prime \prime}\right)\right] .
\end{aligned}
$$

The functions $f_{i}, \theta_{i}, \phi_{i}(i \geq 1)$ are obtained by iteratively solving equations (19) - (22). The approximate solutions for $f(\eta), \theta(\eta)$ and $\phi(\eta)$ are then obtained as

$$
f(\eta) \approx \sum_{m=0}^{\widehat{M}} f_{m}(\eta), \quad \theta(\eta) \approx \sum_{m=0}^{\widehat{M}} \theta_{m}(\eta), \quad \phi(\eta) \approx \sum_{m=0}^{\widehat{M}} \phi_{m}(\eta)
$$

where $\widehat{M}$ is the order of the SLM approximation. Equations (19) - (22) were solved using the Chebyshev spectral collocation method where the unknown functions are approximated using Chebyshev interpolating polynomials at the Gauss-Lobatto points

$$
\xi_{j}=\cos \frac{\pi j}{\widehat{N}}, \quad j=0,1, \ldots, \widehat{N},
$$

where $\widehat{N}$ is the number of collocation points. The physical region $[0, \infty)$ is first transformed into the region $[-1,1]$ using the domain truncation technique in which the problem is solved on the interval $[0, L]$ instead of $[0, \infty)$. This is achieved by using the mapping

$$
\frac{\eta}{L}=\frac{\xi+1}{2}, \quad-1 \leq \xi \leq 1,
$$

where $L$ is the scaling parameter used to invoke the boundary condition at infinity. The unknown functions $f_{i}, \theta_{i}$ and $\phi_{i}$ are approximated at the collocation points by

$$
f_{i}(\xi) \approx \sum_{k=0}^{N} f_{i}\left(\xi_{k}\right) T_{k}\left(\xi_{j}\right), \quad \theta_{i}(\xi) \approx \sum_{k=0}^{N} \theta_{i}\left(\xi_{k}\right) T_{k}\left(\xi_{j}\right), \quad \phi_{i}(\xi) \approx \sum_{k=0}^{N} \phi_{i}\left(\xi_{k}\right) T_{k}\left(\xi_{j}\right), \quad j=0,1, \ldots, \widehat{N},
$$

where $T_{k}$ is the $k$ th Chebyshev polynomial defined as

$$
T_{k}(\xi)=\cos \left[k \cos ^{-1}(\xi)\right] .
$$

The derivatives at the collocation points are represented as

$$
\frac{d^{s} f_{i}}{d \eta^{s}}=\sum_{k=0}^{\widehat{N}} \mathbf{D}_{k j}^{s} f_{i}\left(\xi_{k}\right), \quad \frac{d^{s} \theta_{i}}{d \eta^{s}}=\sum_{k=0}^{\widehat{N}} \mathbf{D}_{k j}^{s} \theta_{i}\left(\xi_{k}\right), \quad \frac{d^{s} \phi_{i}}{d \eta^{s}}=\sum_{k=0}^{\widehat{N}} \mathbf{D}_{k j}^{s} \phi_{i}\left(\xi_{k}\right), \quad j=0,1, \ldots, \widehat{N},
$$


where $s$ is the order of differentiation and $\mathbf{D}=\frac{2}{L} \mathcal{D}$ with $\mathcal{D}$ being the Chebyshev spectral differentiation matrix. Substituting equations (34) - (36) in (19) - (22) leads to the matrix equation

$$
\mathbf{A}_{i-1} \mathbf{X}_{i}=\mathbf{R}_{i-1}
$$

subject to the boundary conditions

$$
\begin{aligned}
& f_{i}\left(\xi_{\widehat{N}}\right)=0, \sum_{k=0}^{\widehat{N}} \mathbf{D}_{\widehat{N} k} f_{i}\left(\xi_{k}\right)=0, \sum_{k=0}^{\widehat{N}} \mathbf{D}_{0 k} f_{i}\left(\xi_{k}\right)=0, \\
& \theta_{i}\left(\xi_{\widehat{N}}\right)=\theta_{i}\left(\xi_{0}\right)=\phi_{i}\left(\xi_{\widehat{N}}\right)=\phi_{i}\left(\xi_{0}\right)=0 .
\end{aligned}
$$

In equation (37), $\mathbf{A}_{i-1}$ is a $(3 \widehat{N}+3) \times(3 \widehat{N}+3)$ square matrix and $\mathbf{X}_{i}$ and $\mathbf{R}_{i}$ are $(3 \widehat{N}+1) \times 1$ column vectors defined by

$$
\mathbf{A}_{i-1}=\left[\begin{array}{lll}
A_{11} & A_{12} & A_{13} \\
A_{21} & A_{22} & A_{23} \\
A_{31} & A_{32} & A_{33}
\end{array}\right], \quad \mathbf{X}_{i}=\left[\begin{array}{c}
\mathbf{F}_{i} \\
\mathbf{\Theta}_{i} \\
\mathbf{\Phi}_{i}
\end{array}\right], \quad \mathbf{R}_{i-1}=\left[\begin{array}{c}
\mathbf{r}_{1, i-1} \\
\mathbf{r}_{2, i-1} \\
\mathbf{r}_{3, i-1}
\end{array}\right],
$$

where

$$
\begin{aligned}
\mathbf{F}_{i} & =\left[f_{i}\left(\xi_{0}\right), f_{i}\left(\xi_{1}\right), \ldots, f_{i}\left(\xi_{\widehat{N}-1}\right), f_{i}\left(\xi_{\widehat{N}}\right)\right]^{T}, \\
\boldsymbol{\Theta}_{i} & =\left[\theta_{i}\left(\xi_{0}\right), \theta_{i}\left(\xi_{1}\right), \ldots, \theta_{i}\left(\xi_{\widehat{N}-1}\right), \theta_{i}\left(\xi_{\widehat{N}}\right)\right]^{T}, \\
\mathbf{\Phi}_{i} & =\left[\phi_{i}\left(\xi_{0}\right), \phi_{i}\left(\xi_{1}\right), \ldots, \phi_{i}\left(\xi_{\widehat{N}-1}\right), \phi_{i}\left(\xi_{\widehat{N}}\right)\right]^{T}, \\
\mathbf{r}_{1, i-1} & =\left[r_{1, i-1}\left(\xi_{0}\right), r_{1, i-1}\left(\xi_{1}\right), \ldots, r_{1, i-1}\left(\xi_{\widehat{N}-1}\right), r_{1, i-1}\left(\xi_{\widehat{N}}\right)\right]^{T}, \\
\mathbf{r}_{2, i-1} & =\left[r_{2, i-1}\left(\xi_{0}\right), r_{2, i-1}\left(\xi_{1}\right), \ldots, r_{2, i-1}\left(\xi_{\widehat{N}-1}\right), r_{2, i-1}\left(\xi_{\widehat{N}}\right)\right]^{T}, \\
\mathbf{r}_{3, i-1} & =\left[r_{3, i-1}\left(\xi_{0}\right), r_{3, i-1}\left(\xi_{1}\right), \ldots, r_{3, i-1}\left(\xi_{\widehat{N}-1}\right), r_{3, i-1}\left(\xi_{\widehat{N}}\right)\right]^{T}, \\
A_{11} & =\mathbf{a}_{1, i-1} \mathbf{D}^{2}+\mathbf{a}_{2, i-1} \mathbf{D}, A_{12}=-\mathbf{I}, A_{13}=-N \mathbf{I} \\
A_{21} & =\mathbf{b}_{4, i-1} \mathbf{D}^{2}+\mathbf{b}_{5, i-1} \mathbf{D}+\mathbf{b}_{6, i-1} \mathbf{I}, A_{22}=\mathbf{b}_{1, i-1} \mathbf{D}^{2}+\mathbf{b}_{2, i-1} \mathbf{D}+\mathbf{b}_{3, i-1} \mathbf{I}, \\
A_{23} & =D_{f} \mathbf{D}^{2}, A_{31}=\mathbf{c}_{4, i-1} \mathbf{D}^{2}+\mathbf{c}_{5, i-1} \mathbf{D}+\mathbf{c}_{6, i-1} \mathbf{I}, \\
A_{32} & =\mathbf{c}_{1, i-1} \mathbf{D}^{2}+\mathbf{c}_{2, i-1} \mathbf{D}+\mathbf{c}_{3, i-1} \mathbf{I}, A_{33}=S_{r} \mathbf{D}^{2} .
\end{aligned}
$$

In the above definitions, $\mathbf{a}_{k, i-1}, \mathbf{b}_{k, i-1}, \mathbf{c}_{k, i-1}(k=1,2, . ., 6)$ are diagonal matrices of size $(\widehat{N}+$ $1) \times(\widehat{N}+1)$ and $\mathbf{I}$ is an identity matrix of size $(\widehat{N}+1) \times(\widehat{N}+1)$. After modifying the matrix system (37) to incorporate boundary conditions (38) - (37), the solution is obtained as

$$
\mathbf{X}_{i}=\mathbf{A}_{i-1}^{-1} \mathbf{R}_{i-1} .
$$

Equations (9) - (12) were further solved numerically using the Matlab bvp4c routine and a shooting technique comprising the Runge-Kutta method of four slopes and the Newton-Raphson method. In solving the boundary value problem by the shooting method, the appropriate ' $\infty$ ' was determined through actual computations and differs for each set of parameter values. 


\subsection{Discussion of smooth cone results}

In the absence of the inertia parameter $\lambda$, Soret and Dufour effects, the non-Darcy problem reduces to that considered by Yih (38) who solved the governing equations using the Keller-box scheme. The problem would also be a special case of the study by Cheng (8) who used a cubic spline collocation method to solve the governing equations. The results from these previous studies are used as a benchmark to test the accuracy of the linearisation method. The heat and mass transfer coefficients are given in Table 1 for different orders of the linearisation method, buoyancy and Lewis numbers. In general, the linearisation method has fully converged to the numerical results at the seventh order for all parameter values.

\begin{tabular}{|c|c|c|c|c|c|c|c|}
\hline & \multirow[t]{2}{*}{$N$} & \multirow[t]{2}{*}{ Le } & \multicolumn{3}{|c|}{$\overline{\text { SLM }}$} & \multirow[t]{2}{*}{$\begin{array}{l}\text { Yih (38) } \\
\text { (3) }\end{array}$} & \multirow[t]{2}{*}{ Cheng (8) } \\
\hline & & & order 3 & order 7 & order 8 & & \\
\hline \multirow{8}{*}{$\frac{N u_{x}}{\sqrt{R a_{x}}}$} & 4 & 1 & 1.5990 & 1.7186 & 1.7186 & 1.7186 & 1.7186 \\
\hline & 4 & 10 & 1.1886 & 1.1795 & 1.1795 & 1.1795 & 1.1794 \\
\hline & 1 & 1 & 1.0869 & 1.0870 & 1.0870 & 1.0869 & 1.0870 \\
\hline & 1 & 10 & 0.9031 & 0.9031 & 0.9031 & 0.9030 & 0.9032 \\
\hline & & & & & & & \\
\hline & 1 & 100 & 0.8141 & 0.8141 & 0.8141 & 0.8141 & 0.8143 \\
\hline & 0 & 1 & 0.7686 & 0.7686 & 0.7686 & 0.7686 & 0.7685 \\
\hline & 0 & 10 & 0.7686 & 0.7686 & 0.7686 & 0.7686 & 0.7685 \\
\hline \multirow{7}{*}{$\frac{S h_{x}}{\sqrt{R a_{x}}}$} & 4 & 1 & 1.5990 & 1.7186 & 1.7186 & 1.7186 & 1.7186 \\
\hline & 4 & 10 & 5.6790 & 5.6980 & 5.6980 & 5.6977 & 5.6949 \\
\hline & 1 & 1 & 1.0869 & 1.0870 & 1.0870 & 1.0869 & 1.0870 \\
\hline & 1 & 10 & 3.8141 & 3.8141 & 3.8141 & 3.8139 & 3.8134 \\
\hline & 1 & 100 & 12.3653 & 12.3653 & 12.3653 & 12.3645 & 12.3377 \\
\hline & 0 & 1 & 0.7686 & 0.7686 & 0.7686 & 0.7686 & 0.7685 \\
\hline & 0 & 10 & 0.7686 & 0.7686 & 0.7686 & 0.7686 & 0.7686 \\
\hline
\end{tabular}

Table 1. Benchmark results for $N u_{x} / R a_{x}^{\frac{1}{2}}$ and $S h_{x} / R a_{x}^{\frac{1}{2}}$ when $\lambda=0.0, n=0.0, R a_{\gamma}=0.0$, $R a_{\xi}=0.0, D_{f}=0.0$ and $S r=0.0$

\begin{tabular}{|c|c|c|c|c|c|c|c|}
\hline & $S r$ & $D_{f}$ & \multicolumn{3}{|c|}{ SLM } & bvp4c & Shooting method \\
\cline { 4 - 6 } & & & order 3 & order 7 & order 8 & & \\
\hline \multirow{2}{*}{\begin{tabular}{c}
$N u_{x}$ \\
\hline$R a_{x}$
\end{tabular}} & 1.5 & 0.03 & 1.550183 & 1.550010 & 1.550010 & 1.550010 & 1.55001 \\
& 0.12 & 1.493268 & 1.493106 & 1.493106 & 1.493106 & 1.49311 \\
& 0.5 & 0.30 & 1.373266 & 1.373121 & 1.373121 & 1.373121 & 1.37312 \\
& 0.1 & 0.60 & 1.170132 & 1.169958 & 1.169958 & 1.169958 & 1.16996 \\
\hline \multirow{2}{*}{$S h_{x}$} & 1.5 & 0.03 & 0.674035 & 0.675657 & 0.675657 & 0.675657 & 0.675658 \\
$\sqrt{R a_{x}}$ & 1.0 & 0.12 & 0.960995 & 0.962038 & 0.962038 & 0.962038 & 0.962039 \\
& 0.5 & 0.30 & 1.251253 & 1.251840 & 1.251840 & 1.251840 & 1.251840 \\
& 0.1 & 0.60 & 1.466009 & 1.466449 & 1.466449 & 1.466449 & 1.466450 \\
\hline
\end{tabular}

Table 2. Comparison of values of $N u_{x} / R a_{x}^{\frac{1}{2}}$ and $S h_{x} / R a_{x}^{\frac{1}{2}}$ for $\lambda=1.0, N=1.0, n=1.0$, $R a_{\gamma}=0.5, R a_{\xi}=0.5$ and $L e=1.0$ 
Table 2 shows the effects of the Dufour and Soret parameters on the heat and mass transfer coefficients when the other parameters are held constant. The accuracy of the method is compared with the Matlab bvp4c solver and a shooting method. Again, the results demonstrate that the SLM is accurate and converges rapidly to the numerical approximations. Furthermore the results show that the heat transfer rate increases with the Soret effect but decreases with the Dufour parameter. On the other hand, mass transfer decreases with increasing Soret numbers while increasing with Dufour numbers. These findings are consistent with those of Narayana and Sibanda (26) where the heat transfer coefficient was observed to increase with increasing values of the Soret parameter while the mass transfer coefficient decreased with increasing values of the Soret parameter.
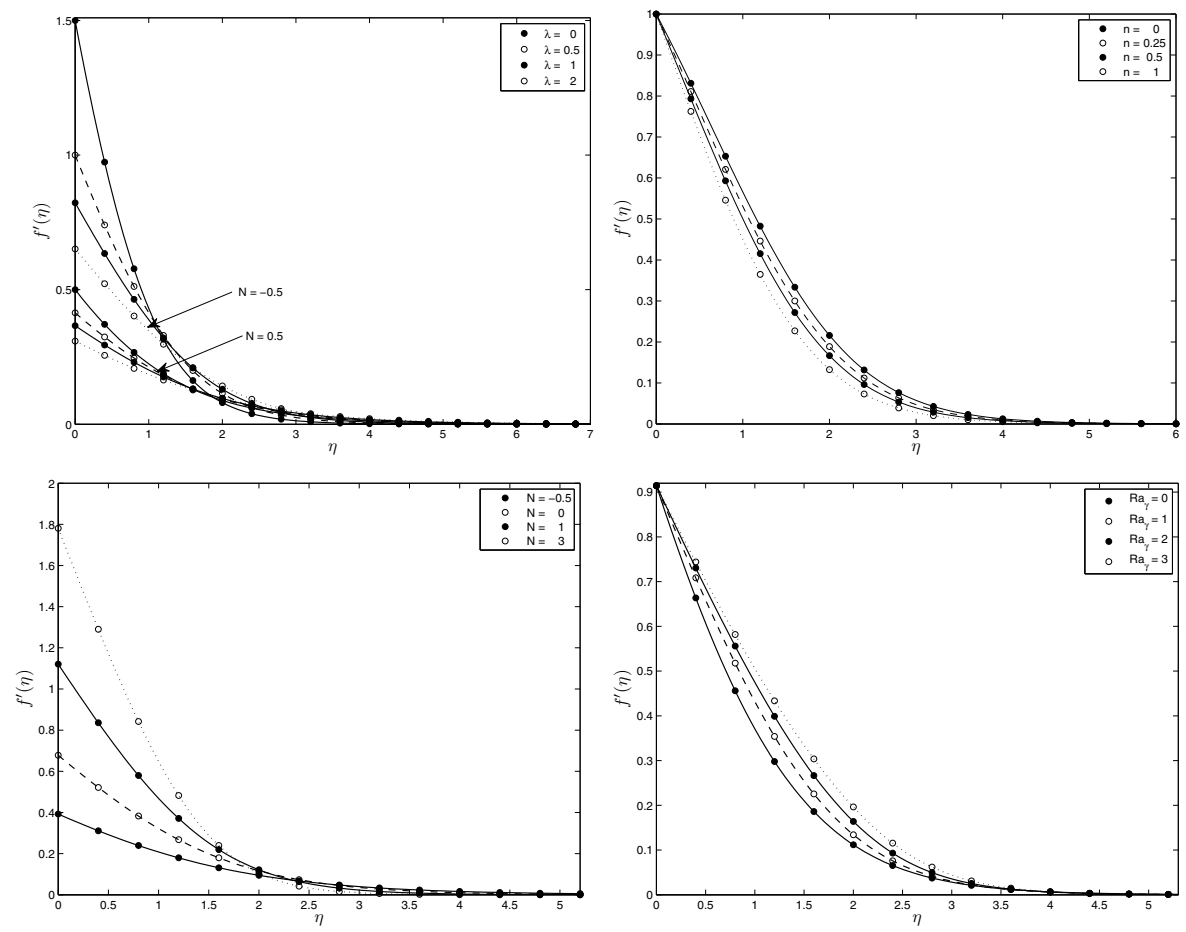

Fig. 2. Effect of (a) inertia parameter $\lambda$, (b) power-law index $n$, (c) buoyancy parameter $N$, and (d) the thermal dispersion parameter $R a_{\gamma}$ on the fluid velocity when $L e=1, S r=0.3$ and $D_{f}=0.2$

Figure 2 shows the effect of (a) the inertia parameter $\lambda$, (b) the power-law index $n$, (c) the buoyancy parameter $N$, and (d) the modified Rayleigh number $R a_{\gamma}$ on the fluid velocity for the inverted cone in a non-Darcy porous medium. Here $N<1$ implies that the concentration buoyancy force is less than the thermal buoyancy force, $N=1$ implies that the buoyancy forces are equal and the case $N>1$ exists when the concentration buoyancy force exceeds the thermal buoyancy force. It is clear that the boundary layer thickness increases with $\lambda, N$ and the Rayleigh number. However, the velocity decreases as the power-law index increases. 

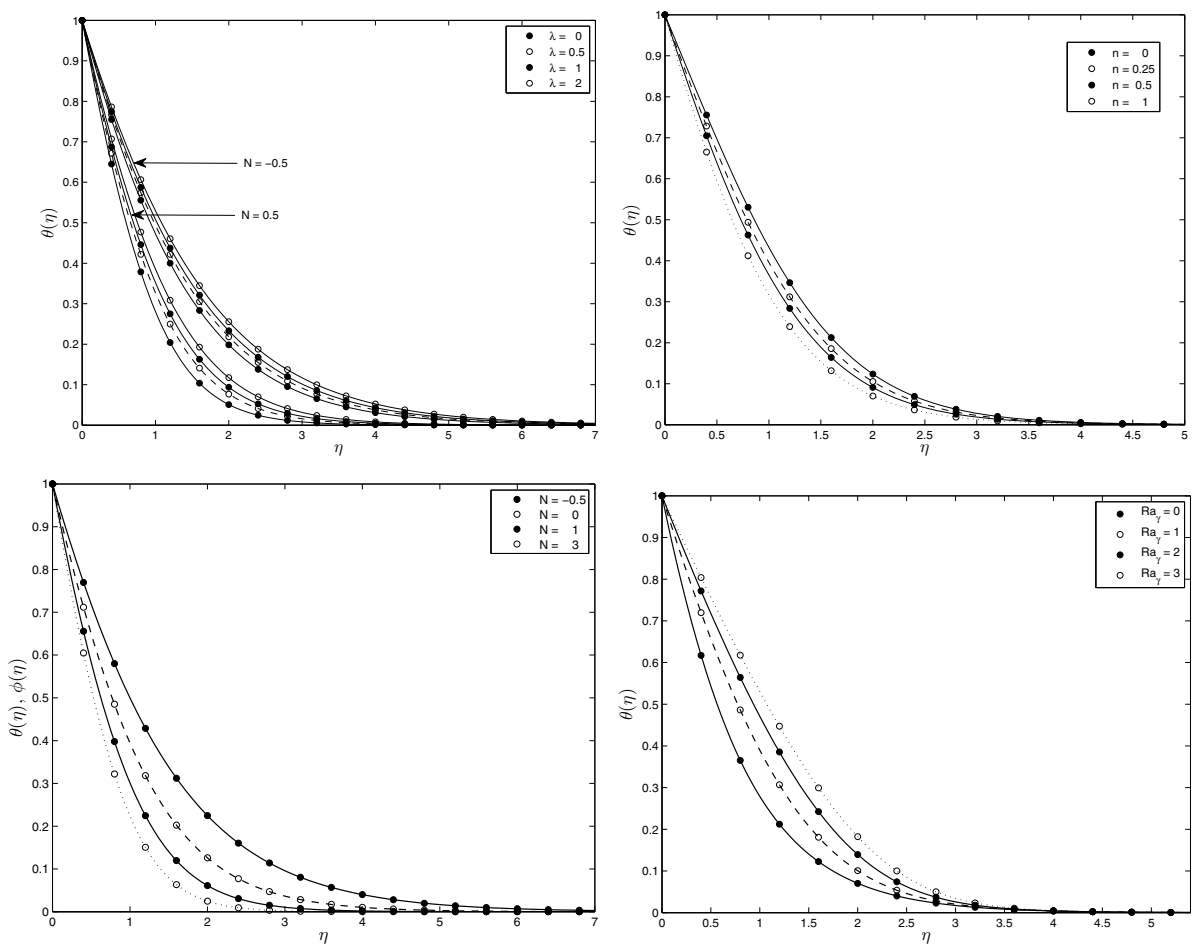

Fig. 3. Effect of (a) inertia parameter $\lambda$, (b) power-law index $n$, (c) the buoyancy parameter $N$, and (d) the thermal dispersion parameter $R a_{\gamma}$ on the temperature profile when $L e=1$, $S r=0.3$ and $D_{f}=0.2$

Figures 3 - 4 show the effects of (a) the inertia parameter $\lambda$, (b) the power-law index $n$, (c) the buoyancy parameter $N$, and (d) the thermal dispersion parameter $R a_{\gamma}$ on the temperature and solute concentration profiles. The temperature profiles decrease with increasing $n$. The concentration profiles increase whereas temperature profile decreases with increasing thermal dispersion parameter.

Figure 5 depicts the variation of the heat transfer rate $N u_{x} R a_{x}^{-1 / 2}$ and the mass transfer rate $S h_{x} R a_{x}^{-1 / 2}$ with Lewis numbers for different values of the Dufour and Soret parameters. For fixed Soret numbers, it is evident that as Le increases, the Nusselt number decreases for any particular value of $D_{f}$. The variation of the Sherwood number with $L e$ for different values of $D_{f}$ is shown in Figure 5(b). Increasing Le enhances the mass transfer rate for any particular value of $D_{f}$. It is also evident that as $D_{f}$ increases the Sherwood number increases for all values of Le.

The variation of the Nusselt and Sherwood numbers with Le and $S r$ when the Dufour number is fixed is shown in Figures 5(c) - 5(d). Increasing Le reduces the Nusselt number for all values of Sr. Conversely, increasing the Soret parameter enhances the Nusselt number. Also, increasing Le contributes to enhancing the mass transfer rate for any particular value of $S r$. On the other hand, increasing $\mathrm{Sr}$ reduces the Sherwood number. 

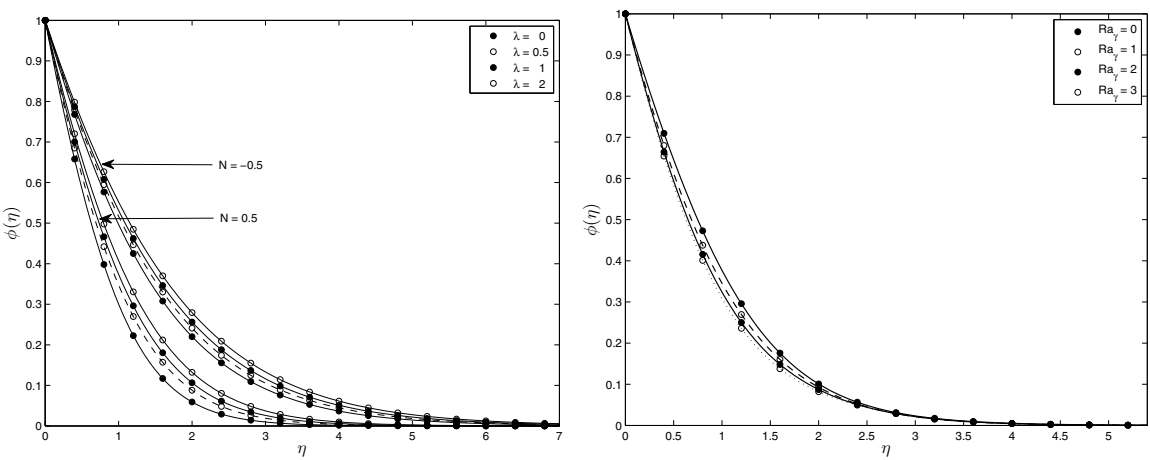

Fig. 4. Effect of (a) inertia parameter $\lambda$, and (b) the thermal dispersion parameter $R a_{\gamma}$ on the concentration profile when $L e=1, S r=0.3$ and $D_{f}=0.2$

\section{Flow over a wavy cone in porous media}

In this section we investigate the case of double-diffusive convection in a fluid around an inverted wavy cone. Figure 6 shows the model of the problem investigated. The wavy surface of the cone is described by

$$
y=\sigma^{*}(x)=a^{*} \sin (\pi x / \ell),
$$

where $a^{*}$ is the amplitude of the wavy surface and $2 \ell$ is the characteristic length of the wave. The governing momentum, heat and solute concentration equations can be written in the form

$$
\begin{aligned}
& \frac{\partial u}{\partial y}-\frac{\partial v}{\partial x}=\frac{g K}{v}\left(\beta_{t} \cos (\Omega) \frac{\partial T}{\partial y}+\beta_{t} \sin (\Omega) \frac{\partial T}{\partial x}+\beta_{c} \cos (\Omega) \frac{\partial C}{\partial y}+\beta_{c} \cos (\Omega) \frac{\partial C}{\partial x}\right) \\
& u \frac{\partial T}{\partial x}+v \frac{\partial T}{\partial y}=\alpha\left(\frac{\partial^{2} T}{\partial x^{2}}+\frac{\partial^{2} T}{\partial y^{2}}\right)+\frac{D k}{c_{s} c_{p}}\left(\frac{\partial^{2} C}{\partial x^{2}}+\frac{\partial^{2} C}{\partial y^{2}}\right) \\
& u \frac{\partial C}{\partial x}+v \frac{\partial C}{\partial y}=D\left(\frac{\partial^{2} C}{\partial x^{2}}+\frac{\partial^{2} C}{\partial y^{2}}\right)+\frac{D k}{c_{s} c_{p}}\left(\frac{\partial^{2} T}{\partial x^{2}}+\frac{\partial^{2} T}{\partial y^{2}}\right)
\end{aligned}
$$

subject to boundary conditions

$$
\begin{aligned}
& v=0, T=T_{w}, C=C_{w} \quad \text { on } \quad y=\sigma^{*}(x)=a^{*} \sin (\pi x / \ell), \\
& u=0, T=T_{\infty}, C=C_{\infty} \quad \text { as } y \rightarrow \infty .
\end{aligned}
$$

Here the symbols have their usual meanings. We now use the following non-dimensional variables;

$$
\begin{aligned}
& (X, Y, R, \sigma, a)=\left(x, y, r, \sigma^{*}, a^{*}\right) / \ell, \quad(U, V)=(u, v) \ell / \alpha, \\
& \Theta=\left(T-T_{\infty}\right) /\left(T_{w}-T_{\infty}\right) \text { and } \Phi=\left(C-C_{\infty}\right) /\left(C_{w}-C_{\infty}\right) .
\end{aligned}
$$




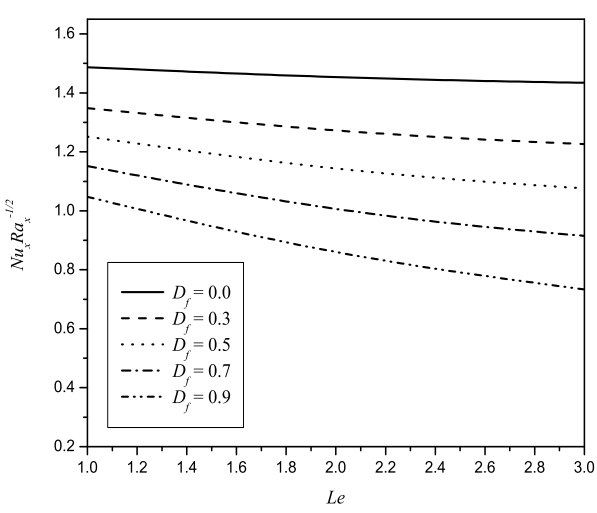

(a)

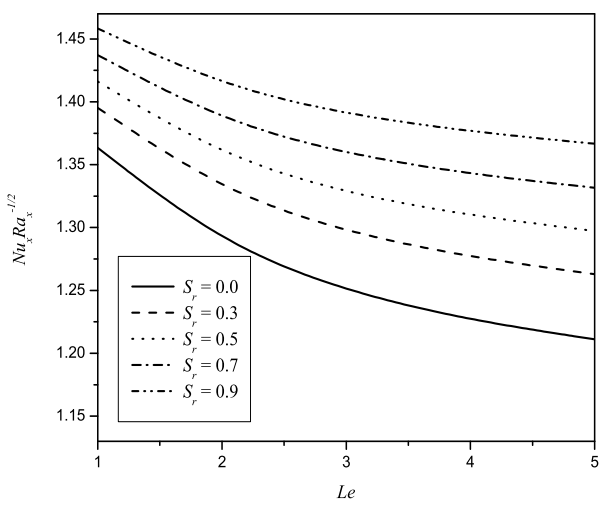

(c)

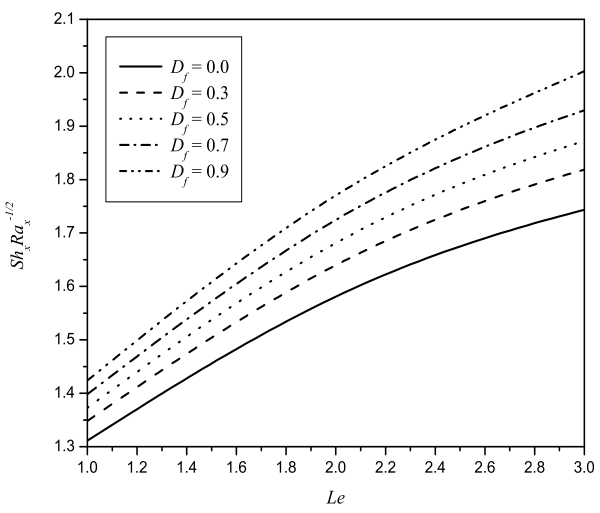

(b)

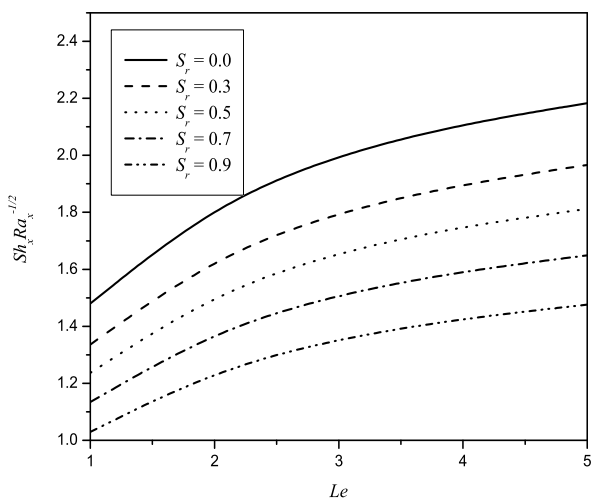

(d)

Fig. 5. The effect of the Dufour and Soret parameters on heat and mass transfers with $\lambda=0.7, n=1, R a_{\gamma}=0.5, R a_{\xi}=0.5, L e=1$ (i) $S r=0.3$ and (ii) $D_{f}=0.2$

The governing equations now become,

$$
\begin{aligned}
& \frac{\partial U}{\partial Y}-\frac{\partial V}{\partial X}=R a\left[\frac{\partial \Theta}{\partial Y}+N \frac{\partial \Phi}{\partial Y}+\tan (\Omega)\left(\frac{\partial \Theta}{\partial X}+N \frac{\partial \Phi}{\partial X}\right)\right], \\
& U \frac{\partial \Theta}{\partial X}+V \frac{\partial \Theta}{\partial Y}=\left(\frac{\partial^{2} \Theta}{\partial X^{2}}+\frac{\partial^{2} \Theta}{\partial Y^{2}}\right)+D_{f}\left(\frac{\partial^{2} \Phi}{\partial X^{2}}+\frac{\partial^{2} \Phi}{\partial Y^{2}}\right), \\
& U \frac{\partial \Phi}{\partial X}+V \frac{\partial \Phi}{\partial Y}=\frac{1}{L e}\left(\frac{\partial^{2} \Phi}{\partial X^{2}}+\frac{\partial^{2} \Phi}{\partial Y^{2}}\right)+S_{r}\left(\frac{\partial^{2} \Theta}{\partial X^{2}}+\frac{\partial^{2} \Theta}{\partial Y^{2}}\right) .
\end{aligned}
$$




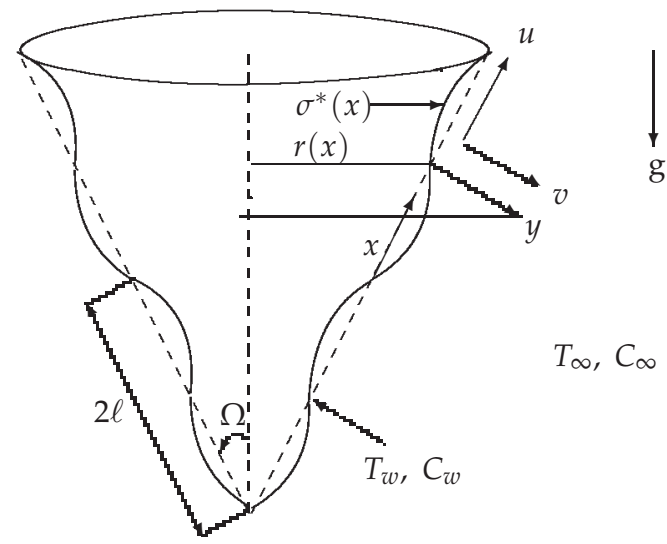

Fig. 6. Schematic sketch of the vertical wavy cone

The parameters appearing above are given by equations (13) - (14). Introducing the stream function $\psi(X, Y)$ defined such that

$$
U=\frac{1}{R} \frac{\partial \psi}{\partial Y} \quad \text { and } \quad V=-\frac{1}{R} \frac{\partial \psi}{\partial X}
$$

equations (60) - (62) can be written in the following form

$$
\begin{aligned}
& \frac{1}{R}\left(\frac{\partial^{2} \psi}{\partial X^{2}}+\frac{\partial^{2} \psi}{\partial Y^{2}}-\frac{R_{X}}{R} \frac{\partial \psi}{\partial X}\right)=R a\left[\frac{\partial \Theta}{\partial Y}+N \frac{\partial \Phi}{\partial Y}+\tan (\Omega)\left(\frac{\partial \Theta}{\partial X}+N \frac{\partial \Phi}{\partial X}\right)\right], \\
& \frac{1}{R}\left(\frac{\partial \psi}{\partial Y} \frac{\partial \Theta}{\partial X}-\frac{\partial \psi}{\partial X} \frac{\partial \Theta}{\partial Y}\right)=\left(\frac{\partial^{2} \Theta}{\partial X^{2}}+\frac{\partial^{2} \Theta}{\partial Y^{2}}\right)+D_{f}\left(\frac{\partial^{2} \Phi}{\partial X^{2}}+\frac{\partial^{2} \Phi}{\partial Y^{2}}\right), \\
& \frac{1}{R}\left(\frac{\partial \psi}{\partial Y} \frac{\partial \Phi}{\partial X}-\frac{\partial \psi}{\partial X} \frac{\partial \Phi}{\partial Y}\right)=\frac{1}{L e}\left(\frac{\partial^{2} \Phi}{\partial X^{2}}+\frac{\partial^{2} \Phi}{\partial Y^{2}}\right)+S_{r}\left(\frac{\partial^{2} \Theta}{\partial X^{2}}+\frac{\partial^{2} \Theta}{\partial Y^{2}}\right),
\end{aligned}
$$

where $R$ is the non-dimensional radius of the cone. The appropriate boundary conditions are

$$
\begin{aligned}
& \psi=0, \Theta=1, \Phi=1 \quad \text { on } \quad Y=\sigma(X)=a \sin (\pi X), \\
& \frac{\partial \psi}{\partial y}=0, \Theta=0, \Phi=0 \quad \text { as } \quad Y \rightarrow \infty .
\end{aligned}
$$

To transform the wavy surface of the cone to a smooth one we introduce the following transformation,

$$
\begin{aligned}
\bar{X} & =X, \\
\bar{Y} R a^{-1 / 2} & =Y-\sigma(X), \\
\bar{\psi} & =R a^{-1 / 2} \psi .
\end{aligned}
$$


Substituting the transformations (70) into equations (64) - (66) and letting $R a \rightarrow \infty$, we obtain the following equations

$$
\begin{aligned}
& \frac{1+\sigma_{\bar{X}}^{2}}{R} \frac{\partial^{2} \bar{\psi}}{\partial \bar{Y}^{2}}=\left[1-\sigma_{\bar{X}} \tan (\Omega)\right]\left(\frac{\partial \Theta}{\partial \bar{Y}}+N \frac{\partial \Phi}{\partial \bar{Y}}\right), \\
& \left(1+\sigma_{\bar{X}}^{2}\right)\left(\frac{\partial^{2} \Theta}{\partial \bar{Y}^{2}}+D_{f} \frac{\partial^{2} \Phi}{\partial \bar{Y}^{2}}\right)=\frac{1}{R}\left(\frac{\partial \bar{\psi}}{\partial \bar{Y}} \frac{\partial \Theta}{\partial \bar{X}}-\frac{\partial \bar{\psi}}{\partial \bar{X}} \frac{\partial \Theta}{\partial \bar{Y}}\right), \\
& \left(1+\sigma_{\bar{X}}^{2}\right)\left(\frac{1}{L e} \frac{\partial^{2} \Phi}{\partial \bar{Y}^{2}}+S_{r} \frac{\partial^{2} \Theta}{\partial \bar{Y}^{2}}\right)=\frac{1}{R}\left(\frac{\partial \bar{\psi}}{\partial \bar{Y}} \frac{\partial \Phi}{\partial \bar{X}}-\frac{\partial \bar{\psi}}{\partial \bar{X}} \frac{\partial \Phi}{\partial \bar{Y}}\right) .
\end{aligned}
$$

We may further simplify equations (70) - (72) by introducing the following transformation

$$
\xi=\bar{X}, \quad \eta=\bar{Y} /\left[\left(1+\sigma_{\xi}^{2}\right) \xi^{1 / 2}\right], \quad \bar{\psi}=R \xi^{1 / 2} f(\xi, \eta), \Theta=\theta(\xi, \eta), \quad \Phi=\phi(\xi, \eta) .
$$

Substituting equation (73) into equations (70) - (72), gives the nonlinear system of differential equations;

$$
\begin{aligned}
& f^{\prime \prime}=\left[1-\sigma_{\xi} \tan (\Omega)\right]\left(\theta^{\prime}+N \phi^{\prime}\right), \\
& \theta^{\prime \prime}+\frac{3}{2} f \theta^{\prime}+D_{f} \phi^{\prime \prime}=\xi\left(f^{\prime} \theta_{\xi}-\theta^{\prime} f_{\xi}\right), \\
& \frac{1}{L e} \phi^{\prime \prime}+\frac{3}{2} f \phi^{\prime}+S_{r} \theta^{\prime \prime}=\xi\left(f^{\prime} \phi_{\xi}-\phi^{\prime} f_{\xi}\right),
\end{aligned}
$$

with boundary conditions

$$
\begin{aligned}
& f(\xi, 0)=0, \quad \theta(\xi, 0)=1, \quad \phi(\xi, 0)=1, \\
& f^{\prime}(\xi, \infty)=0, \quad \theta(\xi, \infty)=0, \quad \phi(\xi, \infty)=0 .
\end{aligned}
$$

The associated local Nusselt and Sherwood numbers are given by

$$
N u_{x}=-R a^{1 / 2} \frac{\xi^{1 / 2} \theta^{\prime}(\xi, 0)}{\left(1+\sigma_{\xi}^{2}\right)^{\frac{1}{2}}} \text { and } S h_{x}=-R a^{1 / 2} \frac{\xi^{1 / 2} \phi^{\prime}(\xi, 0)}{\left(1+\sigma_{\xi}^{2}\right)^{\frac{1}{2}}} .
$$

The mean Nusselt and Sherwood numbers from the leading edge to streamwise position $x$ are given by

$$
\frac{N u_{m}}{R a^{1 / 2}}=-\frac{x}{\ell} \frac{\int_{0}^{\frac{x}{\ell}} \xi^{-1 / 2} \theta^{\prime}(\xi, 0) d \xi}{\int_{0}^{\frac{x}{\ell}}\left(1+\sigma_{\xi}^{2}\right)^{\frac{1}{2}} d \xi}, \frac{S h_{m}}{R a^{1 / 2}}=-\frac{x}{\ell} \frac{\int_{0}^{\frac{x}{\ell}} \xi^{-1 / 2} \phi^{\prime}(\xi, 0) d \xi}{\int_{0}^{\frac{x}{\ell}}\left(1+\sigma_{\xi}^{2}\right)^{\frac{1}{2}} d \xi}
$$

\subsection{Discussion of wavy cone results}

The governing equations (74) - (76) along with the boundary conditions (77), were solved numerically using the Keller-box method (see Keller (16)) for various parameter combinations. Two hundred uniform grid points of step size 0.05 were used in the $\eta$-direction. A uniform grid with 120 nodes was used in the $\xi$ direction. At every $\xi$ grid line, the iteration process is carried out until an accuracy of $10^{-6}$ is achieved for all the variables. The computations carried out are given in Figures 7 to 14 . 
Figure 7 shows the effect of the Dufour number $D_{f}$ on heat and mass transfer for two different values of the amplitude $a$. The effect of increasing the amplitude, on average, is to reduce the heat and mass transfer rates as compared with the limiting case of a smooth cone. Figures 7(c) and 7(d) highlight the same. Figures 7(a) and 7(b) show that for $a=0$ (smooth cone) both $N u_{x} R a^{-1 / 2}$ and $S h_{x} R a^{-1 / 2}$ increase steadily with $\xi$ whereas for the wavy cone (i.e., $a \neq$ $0)$ we observe oscillations in $N u_{x} R a^{-1 / 2}$ and $S h_{x} R a^{-1 / 2}$ over the three complete cycles of undulations from $\xi=0$ to $\xi=6$ having length two. These results represent the nonlinear coupling of the change in fluid velocity and orientation of the gravitation. The results are in agreement with those reported by Cheng (6) and Pop and Na (34). The Dufour number $D_{f}$ reduces $N u_{x} R a^{-1 / 2}$ and $N u_{m} R a^{-1 / 2}$. The opposite is true in the case of $S h_{x} R a^{-1 / 2}$ and $S h_{m} R a^{-1 / 2}$.

The effect of $D_{f}$ on heat and mass transfer is depicted in Figure 8 for two different values of the cone half angle $\Omega$. From $8(\mathrm{c})$ and $8(\mathrm{~d})$ it is clear that increasing the half angle $\Omega$, on average, reduces the heat and mass transfer rates. Figures 8(a) and 8(b) show that there is an increase in oscillations of $N u_{x} R a^{-1 / 2}$ and $S h_{x} R a^{-1 / 2}$ for higher values of $\Omega$. In this case the Dufour number also reduces the heat transfer while enhancing mass transfer.

Figure 9 demonstrates the effect of $D_{f}$ on heat and mass transfer for two different values of buoyancy ratio $N$. It is evident that the buoyancy ratio amplifies heat and mass transfer from the cone. Again, the Dufour number contributes to lowering heat transfer while enhancing mass transfer rates.

The effect of $D_{f}$ on the heat and mass transfer is highlighted for two different values of Lewis numbers in Figure 10. We observe that Le reduces heat transfer whereas the opposite is true in the case of mass transfer. For large values of $L e$, higher values of $D_{f}(\geq 0.5)$ produce negative heat transfer rates indicating that heat diffuses from fluid to the cone in such cases. Figures 10(a) and 10(c) confirm and reinforce the same fact. The effect of Soret number $S_{r}$ on heat and mass transfer for two different values of amplitude $a$ is projected in Figure 11. The decreasing effect of the amplitude $a$ on heat and mass transfer rates observed in this situation also. The Soret number $S_{r}$ contributes to increasing $N u_{x} R a^{-1 / 2}$ and $N u_{m} R a^{-1 / 2}$ while reducing $S h_{x} R a^{-1 / 2} S h_{m} R a^{-1 / 2}$ as can be seen in Figures 11(a) - 11(d).

The effect of $S_{r}$ on heat and mass transfer is shown in Figure 12 for two different values of cone half angle $\Omega$. The fact that $\Omega$ reduces the heat and mass transfer rates is observed in plots 12(a) and 12(d). The Soret number $S_{r}$ has the effect of increasing the heat transfer and reducing the mass transfer for all values of $\Omega$.

Figure 13 shows the effect of $S_{r}$ on heat and mass transfer rates for two different values of the buoyancy ratio $N$. From 13(a) - 13(d) we observe that the buoyancy ratio enhances both heat and mass transfer rates. For selected values of $N, S_{r}$ contributes towards enhancing the heat transfer rate while reducing the mass transfer rate.

The effect of $S_{r}$ on the heat and mass transfer rates is shown in Figure 14 for selected values of the Lewis number Le. It is evident that Le reduces the heat transfer whereas the opposite is true in case of mass transfer. At large values of $L e$ there is a critical value of $S_{r}$ up to which $N u_{x} R a^{-1 / 2}$ and $N u_{m} R a^{-1 / 2}$ increases and beyond this critical value, both $N u_{x} R a^{-1 / 2}$ and $N u_{m} R a^{-1 / 2}$ start to fall as can be more clearly seen in Figures 14(a) and 14(c). From Figures 14(b) - 14(d) we observe that the effect of $S_{r}$ is to reduce the rate of mass transfer from the surface of the wavy cone. 


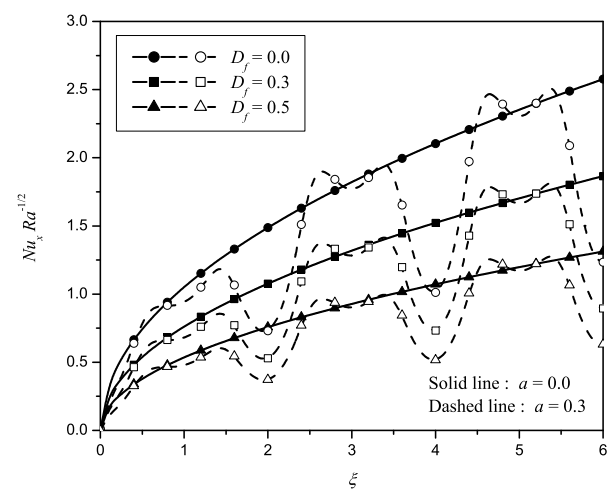

(a)

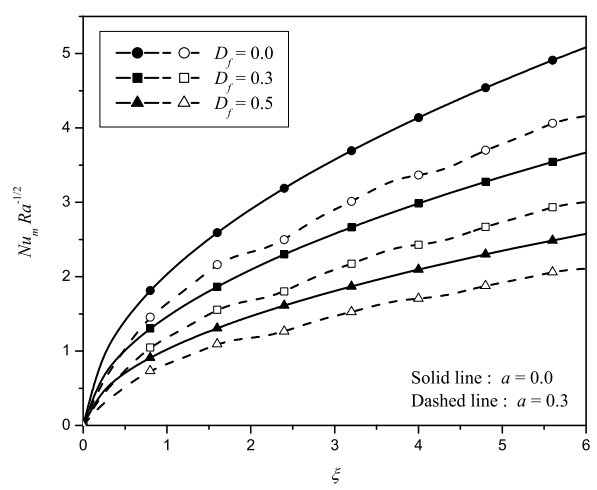

(c)

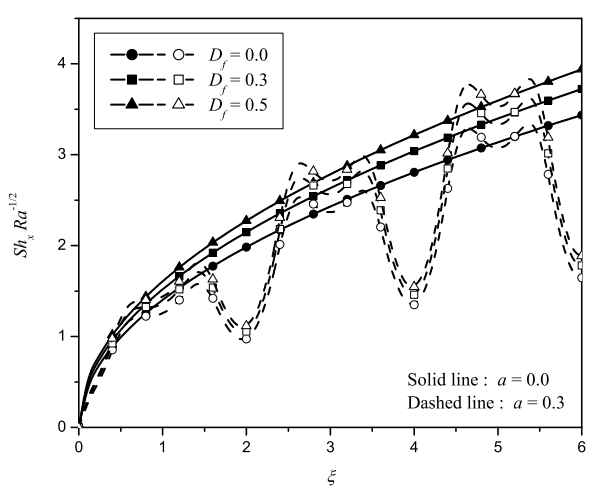

(b)

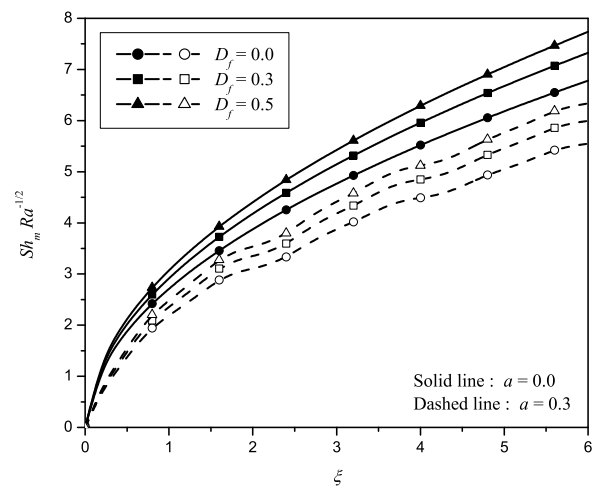

(d)

Fig. 7. Effect of $D_{f}$ on heat and mass transfer with $\Omega=\pi / 9, N=1, L e=2$ and $S_{r}=0.2$ 


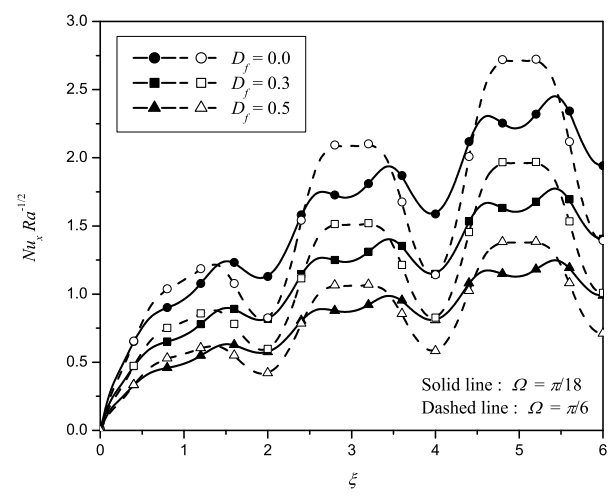

(a)

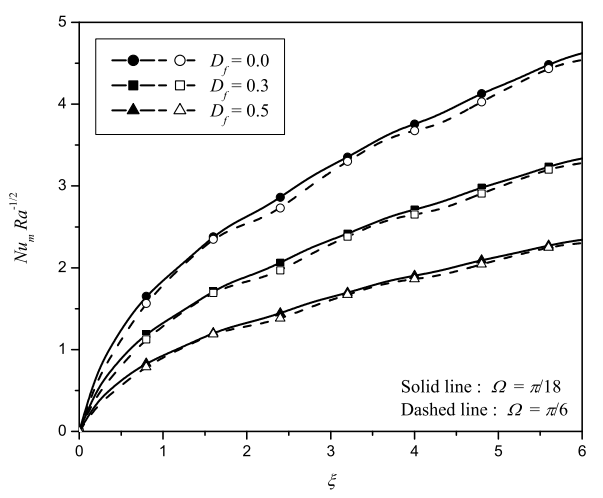

(c)

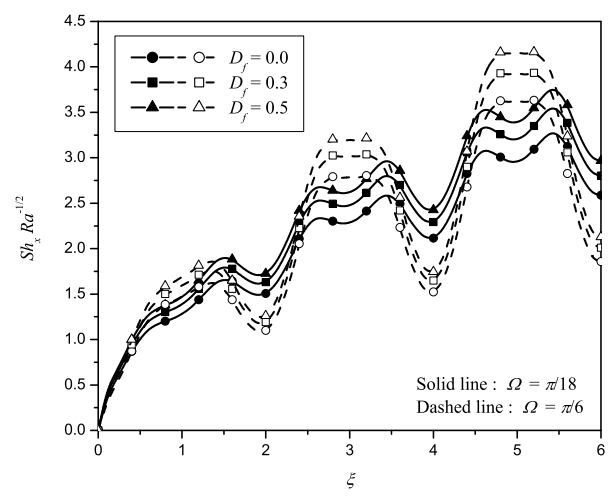

(b)

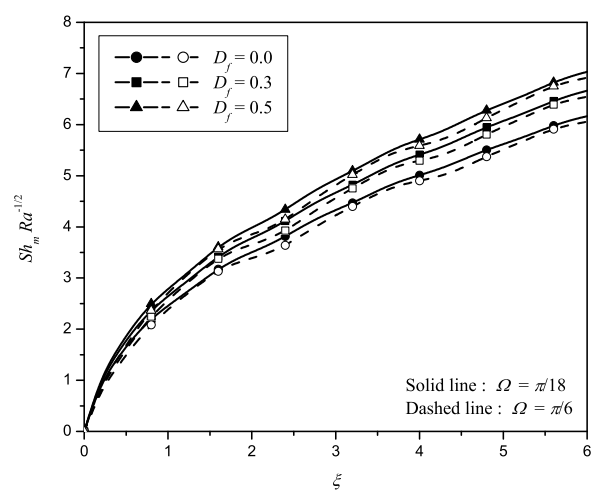

(d)

Fig. 8. Effect of $D_{f}$ on heat and mass transfer with $a=0.2, N=1, L e=2$ and $S_{r}=0.2$ 


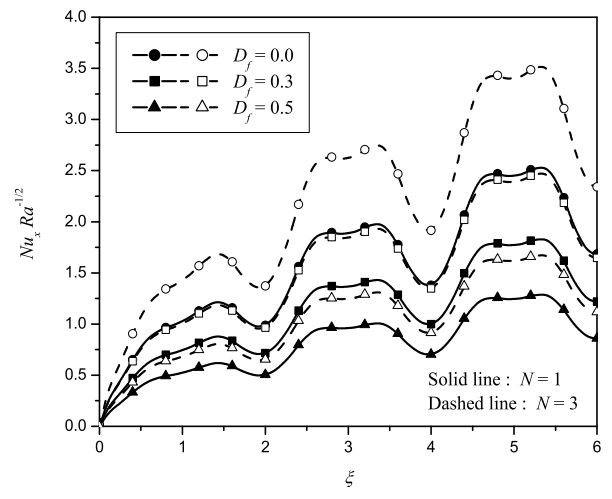

(a)

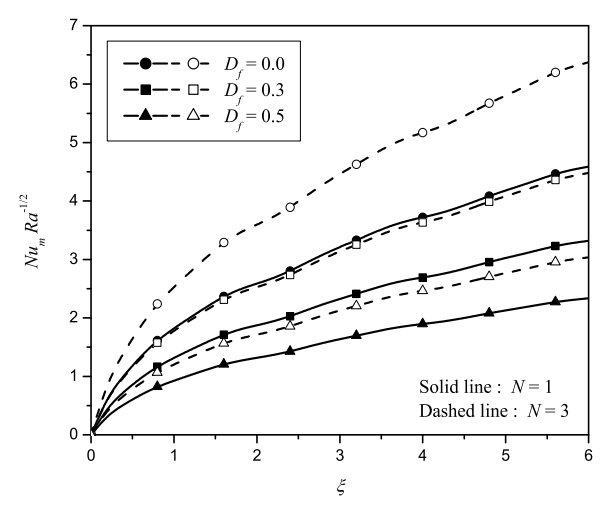

(c)

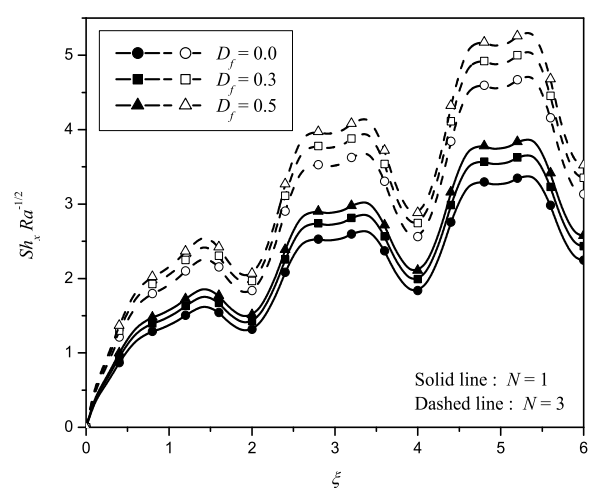

(b)

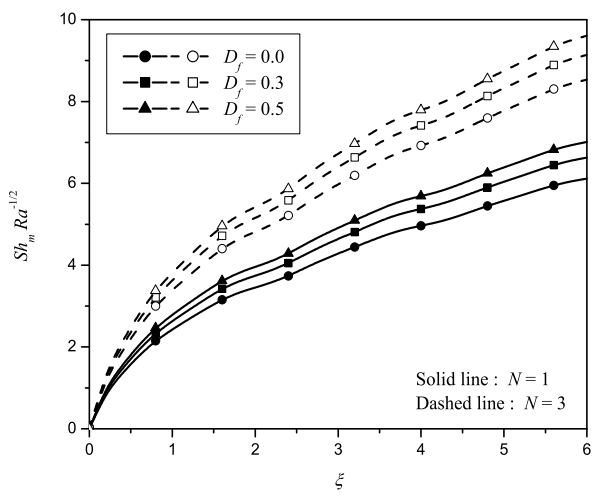

(d)

Fig. 9. Effect of $D_{f}$ on heat and mass transfer with $a=0.2, \Omega=\pi / 9, L e=2$ and $S_{r}=0.2$ 


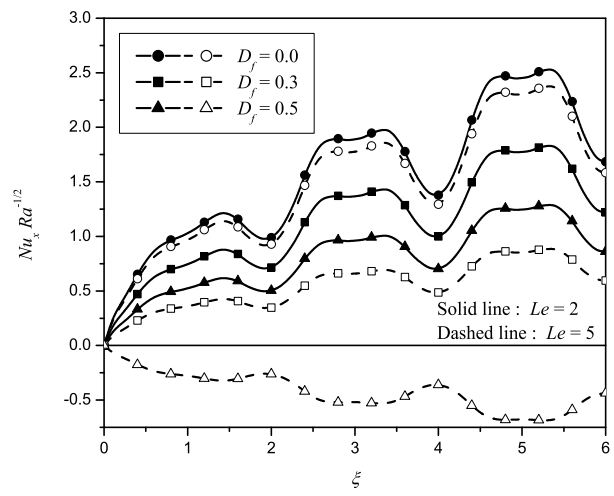

(a)

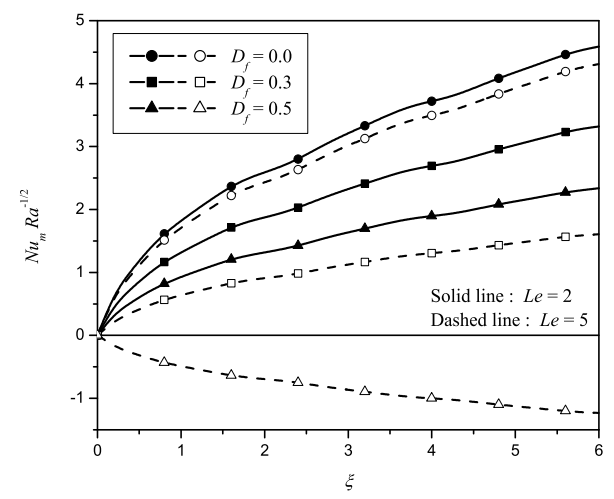

(c)

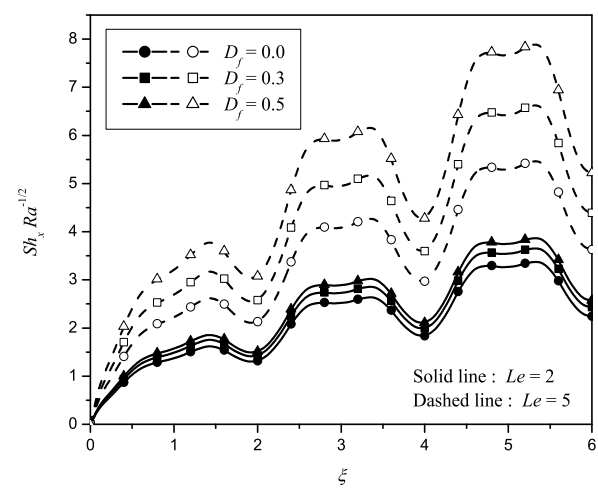

(b)

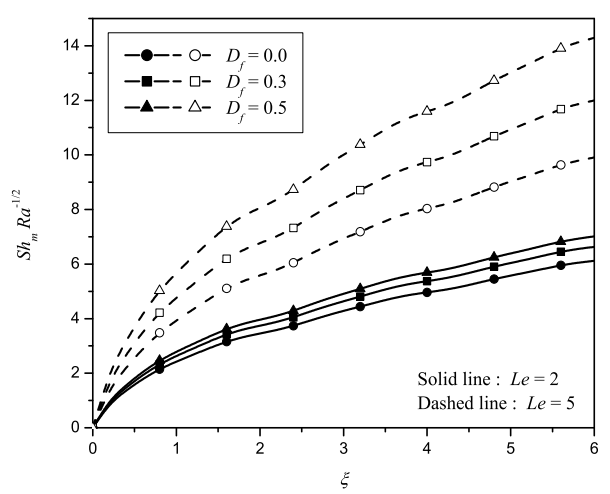

(d)

Fig. 10. Effect of $D_{f}$ on heat and mass transfer with $a=0.2, \Omega=\pi / 9, N=1$ and $S_{r}=0.2$ 


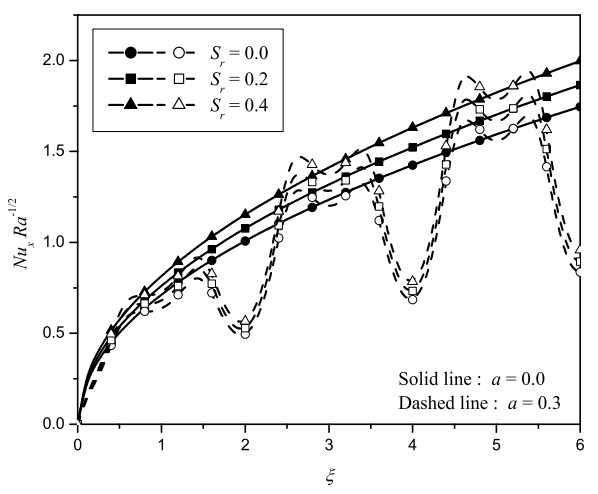

(a)

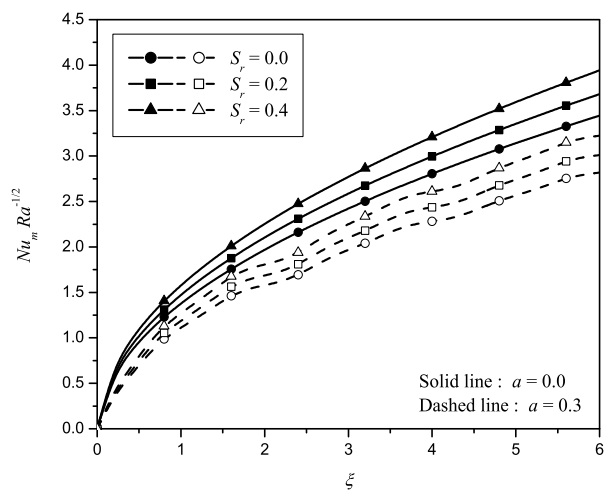

(c)

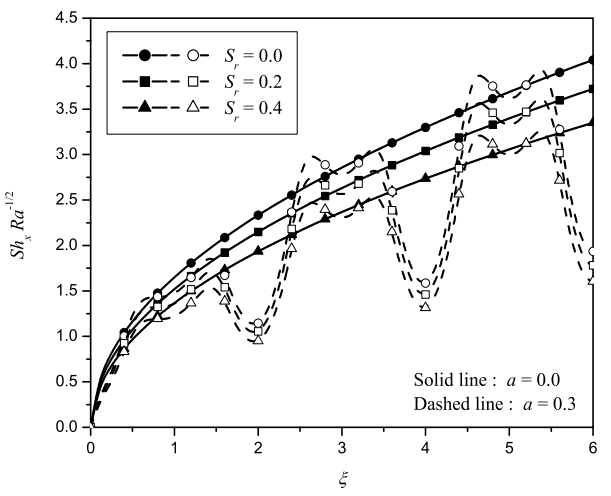

(b)

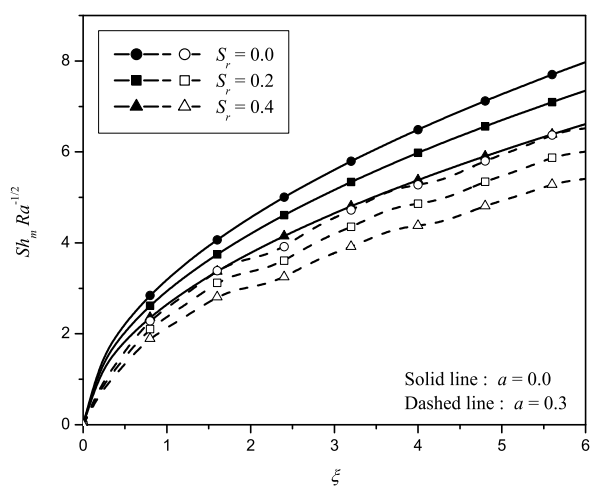

(d)

Fig. 11. Effect of $S_{r}$ on heat and mass transfer with $\Omega=\pi / 9, N=1, L e=2$ and $D_{f}=0.3$ 


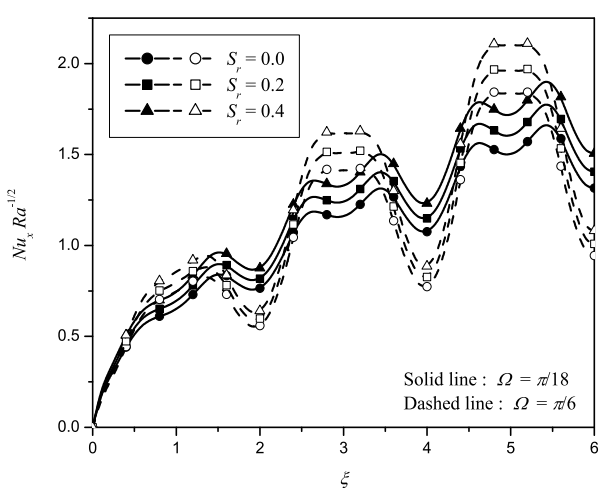

(a)

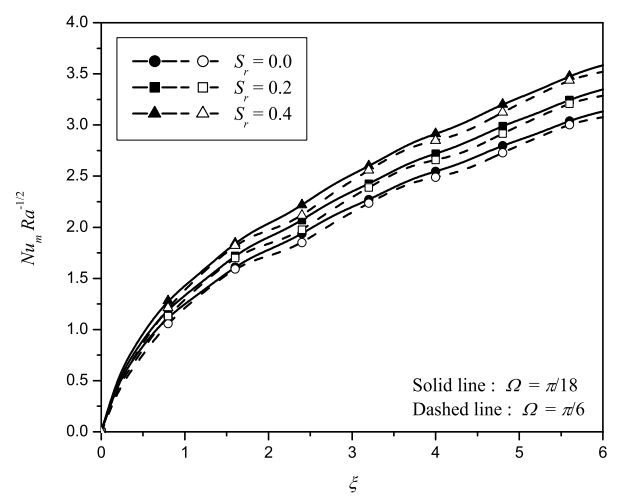

(c)

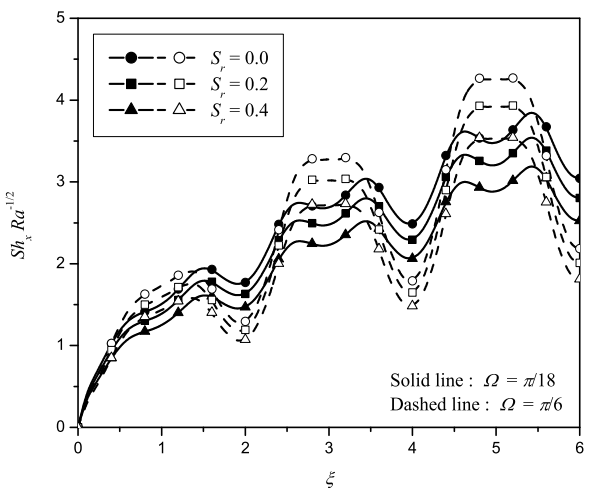

(b)

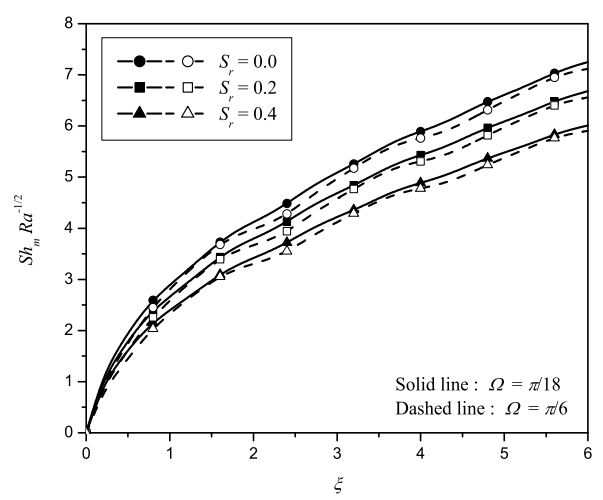

(d)

Fig. 12. Effect of $S_{r}$ on heat and mass transfer with $a=0.2, N=1, L e=2$ and $D_{f}=0.3$ 


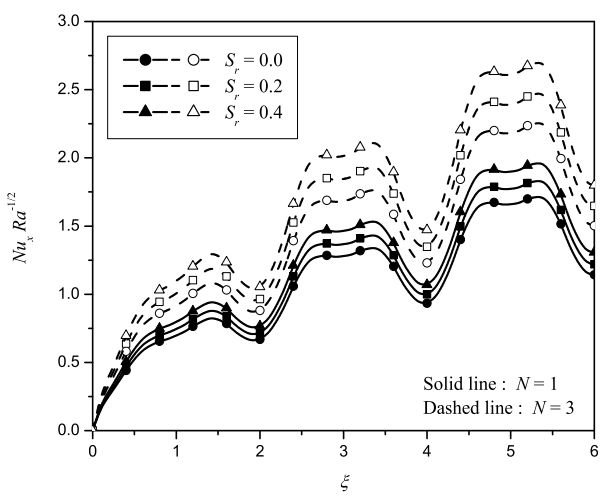

(a)

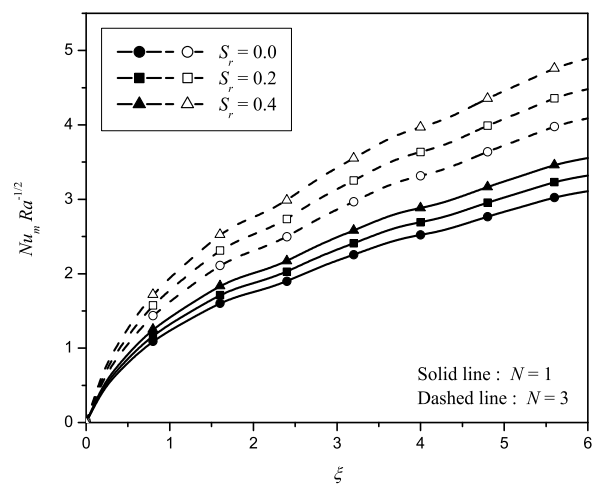

(c)

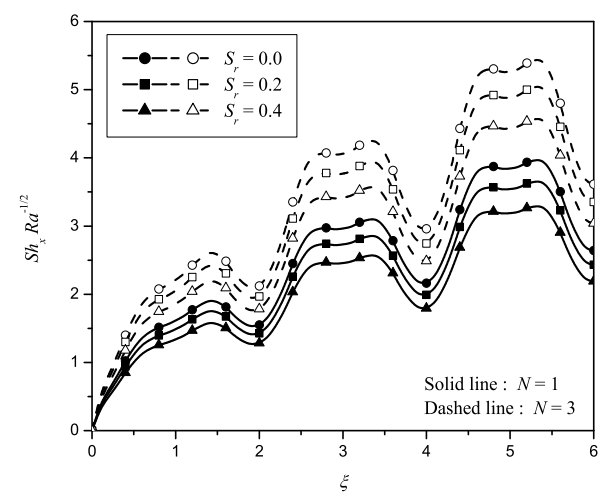

(b)

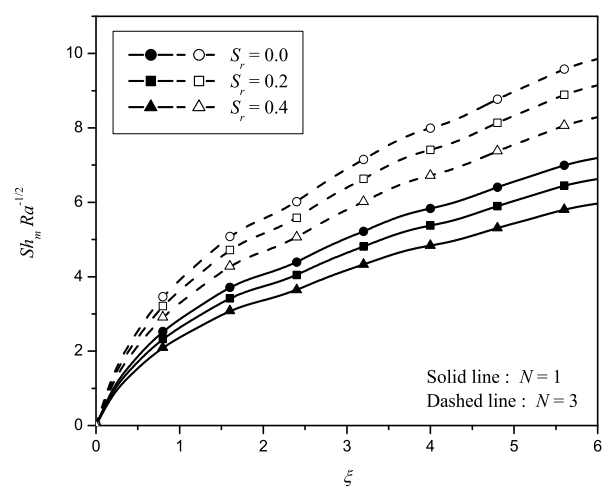

(d)

Fig. 13. Effect of $S_{r}$ on heat and mass transfer with $a=0.2, \Omega=\pi / 9, L e=2$ and $D_{f}=0.3$ 


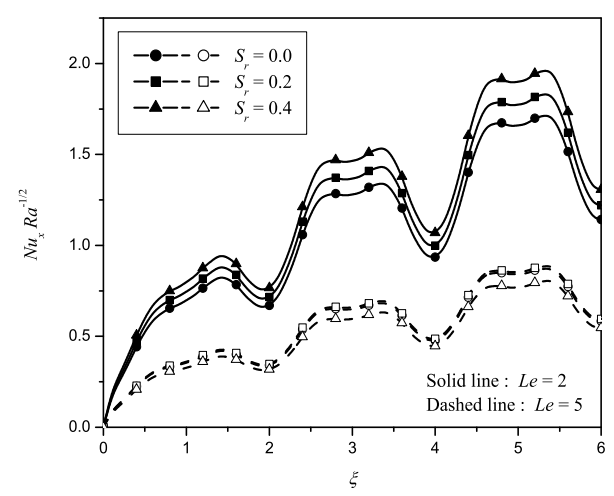

(a)

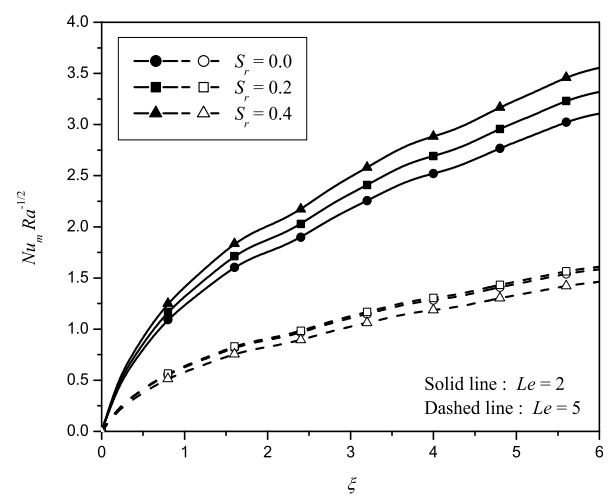

(c)

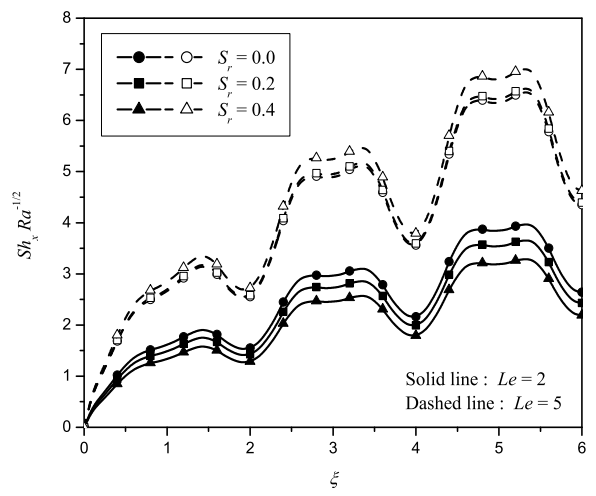

(b)

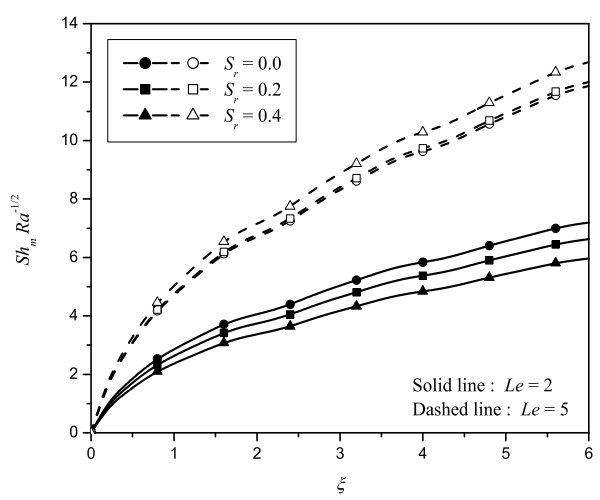

(d)

Fig. 14. Effect of $S_{r}$ on heat and mass transfer with $a=0.2, \Omega=\pi / 9, N=1$ and $D_{f}=0.3$ 


\section{Conclusions}

Double-diffusive convection from inverted smooth and wavy cones in Darcy porous media has been investigated. A similarity analysis is performed to reduce the governing equations to coupled nonlinear differential equations that are solved by using the successive linearisation method (SLM), the Matlab bvp4c, a shooting technique and the Keller-box method.

For the smooth cone the effects of the governing parameters on the velocity, temperature and concentration profiles have been studied. The effects of Dufour and Soret effect on the rate of heat and mass transfer were determined. Comparison between our results and earlier results has been made. The findings suggest that the successive linearisation method is a reliable method for solving nonlinear ordinary differential equations.

In the case of the wavy cone we have studied the effects of cross-diffusion on the heat and the mass transfer rates. From the present study we can see that $D_{f}$ reduces heat transfer and increases mass transfer. The effect of $S_{r}$ is exactly the opposite except at high Lewis numbers when the heat transfer rate increases up to a critical value of $S_{r}$ and then starts decreasing beyond that value.

\section{References}

[1] Akbarzadeh, A. \& P. Manins, P. (1988). Convective layers generated by side walls in solar ponds. Solar Energy, 41(6), pp. 521 - 529.

[2] Alam, M. S.; Rahman, M. M.; Maleque A. \& Ferdows, M. (2006). Dufour and Soret Effects on Steady MHD Combined Free-Forced Convective and Mass Transfer Flow Past a Semi-Infinite Vertical Plate. Thammasat International Journal of Science and Technology, 11, pp. 1 - 12.

[3] Baines, P.G. \& Gill, A. E. (1969). On thermohaline convection with linear gradients, Journal of Fluid Mechanics, 37, pp. 289 - 306.

[4] Chamkha, A. J. \& Khaled, A. A. (1999). Nonsimilar hydromagnetic simultaneous heat and mass transfer by mixed convection from a vertical plate embedded in a uniform porous medium. Numerical Heat Transfer-Part A: Applications, 36, pp. 327 - 344.

[5] Chamkha, A. J. (2001). Coupled heat and mass transfer by natrual convection about a truncated cone in the presence of magnetic field and radiation effects. Numerical Heat Transfer-Part A: Applications, 38, pp. 511 - 530.

[6] Cheng, C. Y. (2000a). Natural convection heat and mass transfer near a wavy cone with constant wall temperature and concentration in a porous medium. Mechanics Research Communications, 27, pp. 613 - 620.

[7] Cheng, C. Y. (2000b). An integral approach for heat and mass transfer by natural convection from truncated cones in porous media with variable wall temperature and concentration. International Communications in Heat and Mass Transfer, 27, pp. 437 - 548.

[8] Cheng, C. -Y. (2009). Soret and Dufour effects on natural convection heat and mass transfer from a vertical cone in a porous medium. International Communications in Heat and Mass Transfer, 36, pp. 1020-1024.

[9] Cheng, P. (1977). Similarity solutions for mixed convection from horizontal impermeable surfaces in saturated porous media. International Journal of Heat Mass Transfer, 20 (9), pp. $893-898$.

[10] Cheng, P. (1985). Natural convection in a porous medium: external flow. Proceedings of the NATO Advanced Study in Natural Convection, Ezmir, Turkey, 1985. 
[11] El-Amin, M.F. (2004). Double dispersion effects on natural convection heat and mass transfer in non-Darcy porous medium. Applied Mathematics and Computation, 156, pp. $1-17$.

[12] Fernando, H. J. S. \& Brandt, A. (1995). Recent advances in double-diffusive convection. Applied Mechanics Reviews, 47(9), pp. C1-C7.

[13] Gaikwad, S. N.; Malashetty, M. S. \& Rama Prasad, K. (2009). An analytic study of linear and nonlinear double-diffusive convection in a fluid saturated anisotropic porous layer with Soret effect. Applied Mathematical Modelling, 33, pp. 3617 - 3635.

[14] Guo, J.; Qin, Y. \& Kaloni, P. N. (1994). Nonlinear stability problem of a rotating doubly diffusive fluid layer. Int. J. Eng. Sci. 32, pp. 1207 - 1219.

[15] Ingham D. B. \& Pop, I. (2002). Transport Phenomenon in Porous Media. Second Ed., Elsevier, Oxford.

[16] Keller, H. B. (1978). Numerical methods in boundary-layer theory. Ann. Rev. Fluid Mech., 10, pp. 417 - 433.

[17] Khanafer, K. \& Vafai, K. (2002). Double-diffusive mixed convection in a lid-driven enclosure filled with a fluid-saturated porous medium. Numerical Heat Transfer. Part A : Applications, 42, pp. 465 - 486.

[18] Lai, F. C. (1990). Coupled heat and mass transfer by natural convection from a horizontal line source in saturated porous medium. International Communications in Heat and Mass Transfer, 17, pp. 489 - 499.

[19] Makukula, Z. G.; Motsa, S. S. \& Sibanda, P. (2010a). On a new solution for the viscoelastic squeezing flow between two parallel plates. Journal of Advanced Research in Applied Mathematics, vol. 2, no. 4, pp. 31-38.

[20] Makukula, Z. G.; Sibanda, P. \& Motsa, S. S. (2010b). A novel numerical technique for two-dimensional laminar flow between two moving porous walls. Mathematical Problems in Engineering, Article ID 528956, 15 pages; doi:10.1155/2010/528-956.

[21] Mansour, M. A.; El-Anssary, N. F. \& Aly, A. M. (2008). Effects of chemical reaction and thermal stratification on MHD free convective heat and mass transfer over a vertical stretching surface embedded in a porous media considering Soret and Dufour numbers. Chemical Engineering Journal, 145, pp. 340-345.

[22] Mortimer, R.G. \& Eyring, H. (1980). Elementary transition state theory of the Soret and Dufour effects. Proceedings of the National Academy of Sciences of the United States of America, vol. 77, no. 4, pp. 1728 - 1731.

[23] Murthy, P. V. S. N. \& Singh, P. (2000). Thermal Dispersion Effects on Non-Darcy Convection over a Cone. International Journal of Computer and Mathematics with Applications, 40, pp. 1433-1444.

[24] Na, T. Y. \& Chiou, J. P. (1979). laminar natural convection over a frustum of a cone. Applied Scientific Research, 35, pp. 409 - 421.

[25] Nakayama, A. \& Hossain, M. A. (1995). An integral treatment for combined heat and mass transfer by natural convection in a porous medium. International Journal of Heat and Mass Transfer, 38, pp. $761-765$.

[26] Narayana, P.A.L. \& Murthy, P.V.S.N. (2008). Soret and Dufour effects on free convection heat and mass transfer from a horizontal flat plate in a Darcy porous medium. International Journal of Heat Tansfer and Mass Transfer, 130, pp. 104504-1-104504-5.

[27] Narayana, P.A.L. \& Sibanda, P. (2010). Soret and Dufour effects on free convection along a vertical wavy surface in a fluid saturated Darcy porous medium. International Journal of Heat and Mass Transfer, 53, pp. 3030-3034. 
[28] Nield, D.A. (1968). Onset of thermohaline convection in a porous medium. Water Resources Research, 5, pp. 553 - 560.

[29] Nield, D.A. \& Bejan, A. (1992). Convection in Porous Media, Springer-Verlag, New York.

[30] Onsager, L. (1931). Reciprocal Relations in Irreversible Processes. Phys. Rev, 37, pp. 405 426.

[31] Partha, M. K. (2009). Suction/injection effects on thermophoresis particle deposition in a non-Darcy porous meduim under the influence of Soret, Dufour effects. International Journal of heat and mass transfer, 52, pp. 1971-1979.

[32] Pop, I. \& Ingham, D. B. (2001). Convective Heat Transfer, Elsevier.

[33] Pop, I. \& Na, T. Y. (1994). Natural convection of a Darcian fluid about a cone. International Communications in Heat and Mass Transfer, 12, pp. 891 - 899.

[34] Pop, I. \& Na, T. Y. (1995). Natural convection over a frustum of a wavy cone in a porous medium. Mechanics Research Communications, 22, pp. 181 - 190.

[35] Stern, M. E. (1960). The 'salt fountain' and thermohaline convection. Tellus, 12, pp. 172 175.

[36] Stern, M. E. (1969). Collective instability of salt fingers. Journal of Fluid Mechanics, 35, pp. $209-218$.

[37] Sunil; Sharma, A. \& Sharma, R. C. (2006). Effect of dust particles on ferrofluid heated and soluted from below. Int. J. Therm. Sci. 45, pp. 347 - 458.

[38] Yih, K. A. (1999). Coupled heat and mass transfer by free convection over a truncated cone in porous media: VWT/ VWC or VHF/VMF. Acta Mechanica, 137, pp. 83-97. 


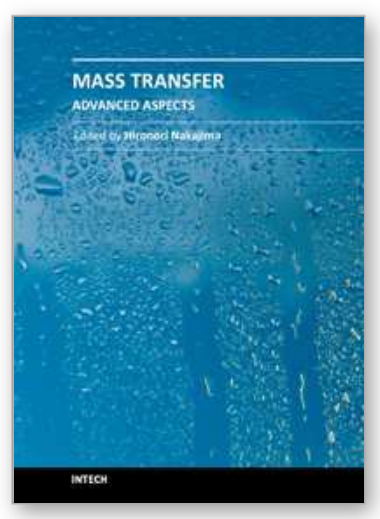

\author{
Mass Transfer - Advanced Aspects \\ Edited by Dr. Hironori Nakajima
}

ISBN 978-953-307-636-2

Hard cover, 824 pages

Publisher InTech

Published online 07, July, 2011

Published in print edition July, 2011

Our knowledge of mass transfer processes has been extended and applied to various fields of science and engineering including industrial and manufacturing processes in recent years. Since mass transfer is a primordial phenomenon, it plays a key role in the scientific researches and fields of mechanical, energy, environmental, materials, bio, and chemical engineering. In this book, energetic authors provide present advances in scientific findings and technologies, and develop new theoretical models concerning mass transfer. This book brings valuable references for researchers and engineers working in the variety of mass transfer sciences and related fields. Since the constitutive topics cover the advances in broad research areas, the topics will be mutually stimulus and informative to the researchers and engineers in different areas.

\title{
How to reference
}

In order to correctly reference this scholarly work, feel free to copy and paste the following:

Faiz GA Awad, Precious Sibanda and Mahesha Narayana (2011). Heat and Mass Transfer from an Inverted Cone in a Porous Medium with Cross-Diffusion Effects, Mass Transfer - Advanced Aspects, Dr. Hironori Nakajima (Ed.), ISBN: 978-953-307-636-2, InTech, Available from: http://www.intechopen.com/books/masstransfer-advanced-aspects/heat-and-mass-transfer-from-an-inverted-cone-in-a-porous-medium-with-crossdiffusion-effects

\section{INTECH}

open science | open minds

\author{
InTech Europe \\ University Campus STeP Ri \\ Slavka Krautzeka 83/A \\ 51000 Rijeka, Croatia \\ Phone: +385 (51) 770447 \\ Fax: +385 (51) 686166 \\ www.intechopen.com
}

\author{
InTech China \\ Unit 405, Office Block, Hotel Equatorial Shanghai \\ No.65, Yan An Road (West), Shanghai, 200040, China \\ 中国上海市延安西路65号上海国际贵都大饭店办公楼 405 单元 \\ Phone: +86-21-62489820 \\ Fax: $+86-21-62489821$
}


(C) 2011 The Author(s). Licensee IntechOpen. This is an open access article distributed under the terms of the Creative Commons Attribution 3.0 License, which permits unrestricted use, distribution, and reproduction in any medium, provided the original work is properly cited. 